\title{
A TP-LPV-LMI Approach to Control of Tumor Growth
}

\author{
György Eigner and Levente Kovács
}

\begin{abstract}
By using advanced control techniques to control physiological systems sophisticated control regimes can be realized. There are several challenges need to be solved in these approaches, however. Most of the time, the lack of information of the internal dynamics, the nonlinear behavior of the system to be controlled and the variabilities coming from that simple fact that people are different and their specifics vary in time makes the control design difficult. Nevertheless, the use of appropriate methodologies can facilitate to find solutions to them. In this study, our aim is to introduce different techniques and by combining them we show an effective way for control design with respect to physiological systems. Our solution stands on four pillars: transformation of the formulated model into control oriented model (COM) form; use the COM for linear parameter varying (LPV) kind modeling to handle unfavorable dynamics as linear dependencies; tensor product modeling (TPM) to downsize the computational costs both from modeling and control design viewpoint; and finally, using linear matrix inequalities (LMI) based controller design to satisfy predefined requirements. The occurring TP-LPV-LMI controller is able to enforce a given, nonlinear system to behave as a selected reference system. In this study, the detailed control solution is applied for tumor growth control to maintain the volume of the tumor.
\end{abstract}

Keywords Tumor growth control $\cdot$ Linear parameter varying $\cdot$ Tensor product model transformation

This project has received funding from the European Research Council (ERC) under the European Union's Horizon 2020 research and innovation programme (grant agreement No 679681).

\footnotetext{
G. Eigner · L. Kovács (凶)

Physiological Controls Research Center, Research, Innovation and Service Center of Óbuda University, Budapest, Hungary

e-mail: kovacs@uni-obuda.hu

G. Eigner

e-mail: eigner.gyorgy@nik.uni-obuda.hu
} 


\section{Introduction}

The so-called Targeted Molecular Therapies (TMTs) are specific therapeutic opportunities came into focus recently concerning the treatment of cancer. They have been successfully applied as therapeutic agents in treatment of different types of tumor moreover they are under investigation as complementary therapies as well regarding classical treatments such as surgery, chemotherapy, radiotherapy and so on. In general they affect the tumors by inhibiting specific biochemical which are responsible for the growth, spread and/or proliferation of tumor concourses [1, 2]. Due to they block or inhibit the properties of tumors in a specific way more personalized therapies can be achieved [3]. Another aspect why TMTs could be good alternative or complementary to conservative therapies is that they are less harmful them, causing less side effects while the load of the body becomes lower. Further they are able to increase the efficiency of regular therapies as well [1]. In the recent times many biochemical pathways have been discovered serving as a basis for TMT developments. The most commonly applied are the apoptosis inducers (facilitating the self-destruction of tumor cells), gene expression inhibitors (decreasing the protein expression in tumor cells which can be useful for the goals of tumor cells), signal transmission inhibitors (inhibiting the biochemical signaling capabilities of tumors) and anti-angiogenic therapies [3].

In this study we consider angiogen inhibition as the basis of therapy. Angiogen inhibitors affect the vascular endothelial growth factor (VEGF) which facilitates the proliferation concerning to the formation of new blood vessels. The process is essential for cancer concourses in order to get enough nutrients needed for further growing after the size of them reach a given volume (diffusion barrier) [4]. The tumors produce VEGF to facilitate the formation of new blood vessels through which the tumor populations can be supported with oxygen and nutrients. The inhibition of this pathway leads to insufficient nutrition from the tumor point of view and after time causing the "starvation" of the tumor $[3,5]$. The way of how this therapy works makes it an excellent therapeutic target and by combining with control engineering methodologies more optimized drug administration can be realized for a better treatment outcome [6]. Bevacizumab is one of the applied anti-angiogen TMT kind drugs considered in this study as well [4].

From the applicable control methods viewpoint several issues need to be overbridged during the design. Alike well-known physiological control problems such as anesthesia or diabetes [7-11], the control of tumor growth is challenging. Several unfavorable effects need to be considered for example the nonlinear behavior of the phenomena to be controlled, the cross-effects, model and parameter uncertainties or even time-delay. Although, it is possible to find solutions suitable with respect to given requirements [6, 12-16].

In this study we investigate a complex controller design approach that involves techniques from the latest developments of control engineering. We alloy the Linear Parameter Variability (LPV) modeling technique with Linear Matrix Inequality (LMI) based controller design both formulated accordingly by Tensor Product (TP) 
model transformation. The LPV framework is a useful technique by which model and parameter uncertainties can be handled moreover it allows the use of linear control design techniques without exact linearization [17-19]. In the recent decades the LMI based control design regarding the LPV framework became well-established especially with respect to state- and output-feedback kind controls. Most of the LMI theorems are based on the laws of Lyapunov [17, 20, 21]. The TP framework is extremely useful because of its ability to make the previously mentioned methods less conservative and the specific properties of the TP form representation makes the LMI based design easier [22-24]. The study investigates a specific TP-LPV-LMI kind controller class regarding tumor growth control. These controllers have many favorable properties for example the uncertainties and nonlinearities can be encapsulated into the model structure leading an "intuitive" robust behavior of the control structure against sort of issues. Moreover the qualitative and quantitative control requirements can be formulated as LMIs through which the designed controller is able to satisfy them $[17,20]$.

During our research state-feedback kind control has applied which requires internal information regarding the state variables during operation. In order to deal with the problem we applied an LPV based Extended Kalman Filter (EKF) solution as an estimator of the state variables. The designed EKF is able to provide the necessary information even though the circumstances [25].

This study is structured in the following way. First we introduce the modeling parts of the research concern to the applied nonlinear tumor growth model, reference model and developed qLPV models. After the controller design is presented including the TP model transformation and the applied LMIs. Then our results are showed and discussed. Finally, we conclude our research and formulate some hints regarding our future work.

\section{Modeling Assumptions}

\subsection{Investigated Tumor Growth Model}

We have applied the modified version of the extended Hahnfeldt-model in this study in accordance with the research goals $[6,16,26]$. The extended model takes into account the absorption dynamics of the inhibitor as well as it is described by (3).

The model has three ordinary differential equations [6]:

$$
\begin{gathered}
\dot{z}_{1}(t)=-\lambda_{1} z_{1}(t) \ln \left(\frac{z_{1}(t)}{z_{2}(t)}\right), \\
\dot{z}_{2}(t)=b z_{1}(t)-d z_{1}^{2 / 3}(t) z_{2}(t)-\eta z_{2}(t) z_{3}(t), \\
\dot{z}_{3}(t)=-\lambda_{3} z_{3}(t)+u(t) .
\end{gathered}
$$


The model consists of three state variables: the tumor volume $z_{1}(t)\left[\mathrm{mm}^{3}\right]$, the supporting vasculature volume $z_{2}(t)\left[\mathrm{mm}^{3}\right]$ and the inhibitor serum level $z_{3}(t)[\mathrm{mg} / \mathrm{kg}]$, respectively. The considered measurable output is the tumor volume $z_{1}(t)\left[\mathrm{mm}^{3}\right]$. The following parameter set has been applied during our investigations: $\lambda_{1}=0.1921$ $1 /$ day, $b=5.8511 /$ day, $d=0.008711 /\left(\mathrm{mm}^{2}\right.$ day $), \eta=0.66 \mathrm{~kg} /(\mathrm{mg}$ day $)$ based on [26]. The $\lambda_{3}=1.31$ 1/day clearance rate belongs to the assumed inhibitor (endostatin) [26].

One has to emphasize that the model has a crucial limitation regarding numerical stability and the border of feasibility. When $z_{1}$ and $z_{2}$ state variables are approaching zero the $\ln \left(\frac{z_{1}(t)}{z_{2}(t)}\right)$ part of (1) tend to $0 / 0$ kind singularity that should be avoided during operation.

As it was proven in [14, 16] by transforming the model the aforementioned issue can be converted into a more suitable form. The $z_{1,2}(t)$ state variables can be transformed and new state variables can be introduced as $x_{1}(t)=\ln \left(z_{1}(t)\right)$, $x_{2}(t)=\ln \left(z_{2}(t)\right)$. The third state variable is linear and not necessary to be transformed so that is $x_{3}(t)=z_{3}(t)$. The new model equations of the extended transformed model of Hahnfeldt can be written in the following way [16]:

$$
\begin{gathered}
\dot{x}_{1}(t)=-\lambda_{1} x_{1}(t)+\lambda_{1} x_{2}(t), \\
\dot{x}_{2}(t)=b e^{x_{1}(t)-x_{2}(t)}-d e^{2 x_{1}(t) / 3}-\eta x_{3}(t), \\
\dot{x}_{3}(t)=-\lambda_{3} x_{3}(t)+u(t) .
\end{gathered}
$$

In order to map the operating domain of the state variables we have to examine (1)(3) as first step. There is a limitation regarding $z_{1}$ and $z_{2}$. The nontrivial equilibrium of the model can be calculated by rearranging (1)-(3) equations. The assumption of permanent inhibitor level $z_{3}(t) \equiv z_{3, \infty}$ leads to the following results $[6,27]$ :

$$
\begin{aligned}
& z_{1, \infty}=z_{2, \infty}=\left(\frac{b-\eta z_{3, \infty}}{d}\right)^{3 / 2}, \\
& z_{1, \text { max }}=z_{2, \text { max }}=\left(\frac{b}{d}\right)^{3 / 2} \leftrightarrow z_{3, \infty}=\frac{1}{\lambda_{3}} u_{\infty} \equiv 0 .
\end{aligned}
$$

Equation(7) shows that the operating domain of $z_{1}$ and $z_{2}$ original state variables are $z_{1}(t), z_{2} \in\left(0,(b / d)^{3 / 2}\right]\left[\mathrm{mm}^{3}\right]$. In this study we assumed $z_{1}(t), z_{2} \in$ $\left[1,(b / d)^{3 / 2}\right]\left[\mathrm{mm}^{3}\right]$. This corresponds to the physiological fact that by using only the anti-angiogenic therapy the tumor cannot be totally eliminated moreover this is in conjunction with our previous findings [6]. The numerical instability can also be avoided in this way. Therefore the operating domain of the transformed state variables becomes $x_{1}, x_{2} \in\left[\ln (1), \ln \left((b / d)^{3 / 2}\right)\right]=[0,9.7648]$.

Accordingly, the goal of the control can be predefined as $x_{1}=x_{2} \rightarrow 0$ when $t \rightarrow t_{\text {end }}$ which is equivalent to $z_{1}=z_{2} \rightarrow 1$ when $t \rightarrow t_{\text {end }}$. With other words, the 
"numerical goals" of the control related to the final values of the states are $x_{1,2, \infty} \equiv 0$ and $z_{1,2, \infty} \equiv 1$.

It should be noted that the extended transformed model is applied for the controller design-moreover, the EKF is also based on the extended transformed model.

\subsection{Control Oriented Model Form}

A given state space model can be written in control oriented model form [17, 28]. That means the state variables of the model are transformed by using a shift operation. The transformed model describes the deviation between a given model equilibrium $\mathbf{x}_{\text {equilibrium }}$ or reference trajectory $\mathbf{x}_{\text {ref }}(t)$ and the actual state variables $\mathbf{x}(t)$. Namely, it models the so-called "error dynamics". By assuming that the shifted difference based state variables describe the deviation between $\mathbf{x}_{\text {ref }}(t)$ and $\mathbf{x}(t)$ the transformation is the following: $\Delta \mathbf{x}(t)=\mathbf{x}(t)-\mathbf{x}_{r e f}(t)$. Naturally, the output, input, disturbances and noises should be transformed if they are interpreted during the controller design, namely, $\Delta y(t)=y(t)-y_{\text {ref }}(t), \Delta u(t)=u(t)-u_{\text {ref }}(t)$, $\Delta d(t)=d(t)-d_{\text {ref }}(t), \Delta n(t)=n(t)-n_{\text {ref }}(t)$.

In case of state-feedback kind controller, the control goal is to enforce the state variables to reach zero. This cannot be directly used in this case. However, by applying the control oriented model form in case of state-feedback the control goal is to enforce the shifted difference based state variables to reach zero-which is equivalent that the state variables of the reference model and the model to be controlled are equal. With other words to eliminate the $\Delta \mathbf{x}(t)$ over time as $\Delta \mathbf{x}(t) \rightarrow \mathbf{0}, t \rightarrow t_{\text {end }}$. This is equivalent with enforcing the model to behave as the selected reference model $\left(\Delta \mathbf{x}(t)=0 \equiv \mathbf{x}(t)=\mathbf{x}_{r e f}(t)\right)$.

\subsection{Development of the Reference Model}

Due to only $x_{1}(t)$ is considered as measurable the reference model has to be based on this state variable. In addition the $x_{1, \text { ref }}(t)$ has to be as simple as possible and it should describe a smoothly decreasing state trajectory. For that reason, we have developed the following reference model:

$$
x_{1, r e f}(t)=e^{(-\xi \cdot t)} \cdot x_{1, r e f}\left(t_{0}\right),
$$

where the numerical value of $\xi$ scalar determines the velocity of decay and $t$ is the time. The initial value $x_{r e f}\left(t_{0}\right)$ is assumed to be known. The initial value can be derived by using measurements (then $x_{1}\left(t_{0}\right)=x_{1, \text { ref }}\left(t_{0}\right)$ ) or estimations (then $\left.\hat{x}_{1}\left(t_{0}\right)=x_{1, \text { ref }}\left(t_{0}\right)\right)$. The reference model will serve as a basis for trajectory planning as it is detailed at the controller design.

In this study $\xi=0.05$ was applied. 


\section{LPV Modeling}

A general parameter varying dynamical system can be described as follows [29]:

$$
\mathcal{S}=(\mathbb{T}, \mathbb{P}, \mathbb{W}, \mathcal{B})
$$

where $\mathcal{S}$ is the LPV system, $\mathbb{T}$ is the time series, $\mathbb{P}$ is the scheduling space, $\mathbb{W}$ is the signal space and $\mathcal{B} \in(\mathbb{W} \times \mathbb{P})^{\mathbb{T}}$ is the behavior of the system $\left((\mathbb{W} \times \mathbb{P})^{\mathbb{T}}\right.$ is the collection of all possible maps from $\mathbb{T}$ to $\mathbb{W} \times \mathbb{P}$ ).

In conformity with [30-32], the general state-space form of an LPV model is the following:

$$
\begin{aligned}
& \dot{\mathbf{x}}(t)=\mathbf{A}(\mathbf{p}(t)) \mathbf{x}(t)+\mathbf{B}(\mathbf{p}(t)) \mathbf{u}(t) \\
& \mathbf{y}(t)=\mathbf{C}(\mathbf{p}(t)) \mathbf{x}(t)+\mathbf{D}(\mathbf{p}(t)) \mathbf{u}(t) \\
& \mathcal{S} \equiv \mathbf{S}(\mathbf{p}(t))=\left[\begin{array}{ll}
\mathbf{A}(\mathbf{p}(t)) & \mathbf{B}(\mathbf{p}(t)) \\
\mathbf{C}(\mathbf{p}(t)) & \mathbf{D}(\mathbf{p}(t))
\end{array}\right] . \\
& \left(\begin{array}{l}
\dot{\mathbf{x}}(t) \\
\mathbf{y}(t)
\end{array}\right)=\mathbf{S}(\mathbf{p}(t))\left(\begin{array}{l}
\mathbf{x}(t) \\
\mathbf{u}(t)
\end{array}\right)
\end{aligned}
$$

The considered vectors and parameter dependent matrices are: $\mathbf{x}(t) \in \mathbb{R}^{n}$ state vector, $\mathbf{u}(t) \in \mathbb{R}^{m}$ input vector, $\mathbf{y}(t) \in \mathbb{R}^{k}$ output vector, $\mathbf{A}(\mathbf{p}(t)) \in \mathbb{R}^{n \times n}$ state matrix, $\mathbf{B}(\mathbf{p}(t)) \in \mathbb{R}^{n \times m}$ input matrix, $\mathbf{C}(\mathbf{p}(t)) \in \mathbb{R}^{k \times n}$ output matrix, $\mathbf{D}(\mathbf{p}(t)) \in \mathbb{R}^{k \times m}$ feed-forward matrix and $\mathbf{S}(\mathbf{p}(t)) \in \mathbb{R}^{(n+k) \times(n+m)}$ system matrix. The matrices in (5) are dependent from the $\mathbf{p}(t) \in \Omega^{R} \in \mathbb{R}^{R}$ parameter vector which consists of the so-called scheduling variables $p_{i}(t)$, namely, $\mathbf{p}(t)=\left[p_{1}(t) \ldots p_{R}(t)\right]^{\top}$. The $\Omega=$ $\left[p_{1, \text { min }}, p_{1, \text { max }}\right] \times\left[p_{2, \text { min }}, p_{2, \text { max }}\right] \times \cdots \times\left[p_{R, \text { min }}, p_{R, \text { max }}\right] \in \mathbb{R}^{R} \quad$ hypercube-a subspace of the $\mathbb{R}^{R}$ real vector space-is characterized by the extremes of the $p_{i}(t)$.

An LPV model inasmuch any of the state variables are involved into the scheduling parameters it is called qLPV model [31].

\section{1 qLPV Model Development}

During the qLPV modeling we use the assumptions of Sect. 2.2. The transformation of the first and third state variables are equivocal—since the (4) and (6) are linear equations.

The transformations can be done as follows:

$$
\begin{aligned}
& \Delta \dot{x}_{1}(t)=\dot{x}_{1}(t)-\dot{x}_{1, r e f}(t)=-\lambda_{1} x_{1}(t)+\lambda_{1} x_{2}(t)- \\
& \left(-\lambda_{1} x_{1, r e f}(t)+\lambda_{1} x_{2, r e f}(t)\right)= \\
& -\lambda_{1}\left(x_{1}(t)-x_{1, r e f}(t)\right)+\lambda_{1}\left(x_{2}(t)-x_{2, r e f}(t)\right)= \\
& -\lambda_{1} \Delta x_{1}(t)+\lambda_{1} \Delta x_{2}(t) .
\end{aligned}
$$




$$
\begin{aligned}
& \Delta \dot{x}_{3}(t)=\dot{x}_{3}(t)-\dot{x}_{3, r e f}(t)= \\
& -\lambda_{3} x_{3}(t)+u(t)-\left(-\lambda_{3} x_{3, r e f}(t)+u_{r e f}(t)\right)= \\
& -\lambda_{3} \Delta x_{3}(t)+\Delta u(t)
\end{aligned}
$$

Due to the exponential functions transformation of (5) it results:

$$
\begin{aligned}
& \Delta \dot{x}_{2}(t)=\dot{x}_{2}(t)-\dot{x}_{2, r e f}(t)= \\
& b e^{x_{1}(t)-x_{2}(t)}-d e^{2 x_{1}(t) / 3}-\eta x_{3}(t) \\
& -\left(b e^{x_{1, r e f}(t)-x_{2, r e f}(t)}-d e^{2 x_{1, r e f}(t) / 3}-\eta x_{3, r e f}(t)\right) .
\end{aligned}
$$

In order to reach the desired qLPV form the following mathematical manipulations can be applied:

$$
\begin{aligned}
& b e^{x_{1}(t)} e^{-x_{2}(t)}-b e^{x_{1, r e f}(t)} e^{-x_{2, r e f}(t)}-0= \\
& b e^{x_{1}(t)} e^{-x_{2}(t)}-b e^{x_{1, r e f}(t)} e^{-x_{2, r e f}(t)}-b e^{x_{1, r e f}(t)} e^{-x_{2}(t)} \\
& +b e^{x_{1, r e f}(t)} e^{-x_{2}(t)}=
\end{aligned} \begin{gathered}
b e^{-x_{2}(t)}\left(e^{x_{1}(t)}-e^{x_{1, r e f}(t)}\right) \cdot 1 \\
-b e^{x_{1, r e f}(t)}\left(e^{-x_{2}(t)}-e^{-x_{2, r e f}(t)}\right) \cdot 1= \\
b e^{-x_{2}(t)} \frac{\left(e^{x_{1}(t)}-e^{x_{1, r e f}(t)}\right)}{\Delta x_{1}(t)} \Delta x_{1}(t) \\
-b e^{x_{1, r e f}(t)} \frac{\left(e^{-x_{2}(t)}-e^{-x_{2, r e f}(t)}\right)}{\Delta x_{2}(t)} \Delta x_{2}(t) \\
-d e^{2 x_{1}(t) / 3}+d e^{2 x_{1, r e f}(t) / 3}= \\
-d\left(e^{2 x_{1}(t) / 3}-e^{2 x_{1, r e f}(t) / 3}\right) \cdot 1= \\
-d \frac{\left(e^{2 x_{1}(t) / 3}-e^{2 x_{1, r e f}(t) / 3}\right)}{\Delta x_{1}(t)} \Delta x_{1}(t) . \\
-\eta x_{3}(t)+\eta x_{3, r e f}(t)=-\eta \Delta x_{3}(t) .
\end{gathered}
$$

From (12) two scheduling variables can be selected based on (14):

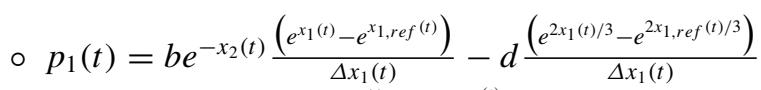

○ $p_{2}(t)=-b e^{x_{1, r e f}(t)} \frac{\left(e^{-x_{2}(t)}-e^{-x_{2, r e f}(t)}\right)}{\Delta x_{2}(t)}$

Accordingly, the transformed $\Delta x_{2}(t)$ state variable becomes:

$$
\Delta \dot{x}_{2}(t)=p_{1}(t) \Delta x_{1}(t)+p_{2}(t) \Delta x_{2}(t)-\eta \Delta x_{3}(t) .
$$

The $p_{1,2}(t)$ has to be investigated from stability point of view since the divisions by $\Delta x_{1,2}(t)$ in both terms may cause numerical instability when $\Delta x_{1,2}(t) \rightarrow 0$. In order to decide what happens when $\Delta x_{1,2}(t) \rightarrow 0$ the L'Hospital's rule [33] can be applied, namely, to calculate the finite final values (if any) of $\left.p_{1}(t)\right|_{\Delta x_{1}(t)=0}$ and $\left.p_{2}(t)\right|_{\Delta x_{2}(t)=0}$. 


$$
\begin{aligned}
& \lim _{\Delta x_{1}(t) \rightarrow 0} p_{1}(t)=\lim _{\Delta x_{1}(t) \rightarrow 0} b e^{-x_{2}(t)} \frac{\left(e^{x_{1}(t)}-e^{x_{1, r e f}(t)}\right)}{\Delta x_{1}(t)} \\
&-d \frac{\left(e^{2 x_{1}(t) / 3}-e^{2 x_{1, r e f}(t) / 3}\right)}{\Delta x_{1}(t)}= \\
&\left(e^{x_{1}(t)}+e^{x_{1, r e f}(t)}\right)\left(\frac{2}{3} d+b e^{2 x_{2}(t)}\right) . \\
& \lim _{\Delta x_{2}(t) \rightarrow 0} p_{2}(t)=\lim _{\Delta x_{2}(t) \rightarrow 0} b e^{-x_{1, r e f}(t)} \frac{\left(e^{x_{2}(t)}-e^{x_{2, r e f}(t)}\right)}{\Delta x_{2}(t)}= \\
&-b e^{x_{1, r e f}(t)}\left(e^{x_{2}(t)}+e^{x_{2, r e f}(t)}\right) .
\end{aligned}
$$

By considering (15) these conditions has to be embedded into the implementation in the following way.

$$
\begin{gathered}
p_{1}(t)=\left\{\begin{array}{cc}
b e^{-x_{2}(t)} \frac{\left(e^{x_{1}(t)}-e^{x_{1, r e f}(t)}\right)}{\Delta x_{1}(t)} & \text { if } \Delta x_{1}(t)=0 \\
-d \frac{\left(e^{2 x_{1}(t) / 3}-e^{2 x_{1, r e f}(t) / 3}\right)}{\Delta x_{1}(t)}, & \text { if } \Delta x_{1}(t) \neq 0 \\
\left(e^{x_{1}(t)}+e^{x_{1, r e f}(t)}\right)\left(\frac{2}{3} d+b e^{2 x_{2}(t)}\right), & \text { if } \Delta x_{2}(t) \neq 0
\end{array}\right. \\
p_{2}(t)= \begin{cases}b e^{-x_{1, r e f}(t)} \frac{\left(e^{x_{2}(t)}-e^{x_{2, r e f}(t)}\right)}{\Delta x_{2}(t)}, & \text { if } \Delta x_{2}(t)=0 \\
-b e^{x_{1, r e f}(t)}\left(e^{x_{2}(t)}+e^{x_{2, r e f}(t)}\right),\end{cases}
\end{gathered}
$$

The operating domain of $\mathbf{p}(t)$ can be calculated based on (17)-(18) by considering the values of $x_{1,2}(t)$ and $x_{1,2, \text { ref }}(t)$ which may appear during operation. Based on our experiments in the topic we found that the application of $\Omega=\left[p_{1, \min }, p_{1, \max }\right] \times\left[p_{2, \min }, p_{2, \max }\right]=[0,16] \times[4,15]$ domain can be applied, namely $p_{1}(t)=[0, \ldots, 16]$ and $p_{2}(t)=[4, \ldots, 15]$. The reason is to take into account the physiological reasonable minimum and maximum numerical values of functions in (17)-(18).

The LPV model can be written in control oriented model form as with respect to Sect. 2.2 by considering (11), (12) and (15):

$$
\begin{gathered}
\Delta \dot{\mathbf{x}}(t)=\mathbf{A}(\mathbf{p}(t)) \Delta \mathbf{x}(t)+\mathbf{B} \Delta \mathbf{u}(t) \\
\Delta \mathbf{y}(t)=\mathbf{C} \Delta \mathbf{x}(t) \\
\mathbf{S}(\mathbf{p}(t))=\left[\begin{array}{ccc}
\mathbf{A}(\mathbf{p}(t)) & \mathbf{B} \\
\mathbf{C} & 0
\end{array}\right]=\left[\begin{array}{cccc}
-\lambda_{1} & \lambda_{1} & 0 & 0 \\
p_{1}(t) & p_{2}(t) & -\eta & 0 \\
0 & 0 & -\lambda_{3} & 1 \\
1 & 0 & 0 & 0
\end{array}\right]
\end{gathered}
$$




\section{Control Design}

\subsection{TP Modeling and Control}

The TP model transformation is a mathematical tool which is able to convert arbitrary-but appropriately formalized - qLPV model into TP model form. The resulting TP model approximates the qLPV model via the original nonlinear model as well with given accuracy depends on the sampling density applied on $\Omega$. The TP model transformation has been successfully applied in several cases regarding nonlinear control problems (e.g. [23, 28, 34-36]). The TP model transformation can be directly applied on (10) and (19) [17]. The resulting finite element convex polytopic TP model is the following:

$$
\begin{aligned}
& \left(\begin{array}{l}
\dot{\mathbf{x}}(t) \\
\mathbf{y}(t)
\end{array}\right)=\mathbf{S}(\mathbf{p}(t))\left(\begin{array}{l}
\mathbf{x}(t) \\
\mathbf{u}(t)
\end{array}\right) \\
& \mathbf{S}(\mathbf{p}(t))=\mathcal{S} \underset{\mathrm{r}=1}{\mathrm{R}} \mathbf{w}_{r}\left(p_{r}(t)\right)=\mathcal{S} \times_{r} \mathbf{w}(\mathbf{p}(t))
\end{aligned},
$$

where $\mathcal{S}$ core tensor composed of $\mathbf{S}_{i_{1}, i_{2}, \ldots, i_{R}}$ LTI vertices as $\mathcal{S} \in \mathbb{R}^{I_{1} \times I_{2} \times \cdots \times I_{R} \times(n+k) \times(n+m)}$. Each of the $\mathbf{S}_{i_{1}, i_{2}, \ldots, i_{R}}$ terms represents a given LTI system with different parameter set. The $\mathbf{w}_{r}\left(p_{r}(t)\right)$ weighting vector determines which LTI system dominates in the resulting $\mathbf{S}(\mathbf{p}(t))$. The $\mathbf{w}_{r}\left(p_{r}(t)\right)$ weighting vector function is composed of $w_{r, i_{r}}\left(p_{r}(t)\right)\left(i_{r}=1 \ldots I_{R}\right)$ continuous convex weighting functions.

The convexity property holds if the followings are satisfied:

$$
\begin{aligned}
& \forall r, i, p_{r}(t): w_{r, i_{r}}\left(p_{r}(t)\right) \in[0,1] \\
& \forall r, p_{r}(t): \sum_{i=1}^{I_{r}} w_{r, i_{r}}\left(p_{r}(t)\right)=1 .
\end{aligned}
$$

By applying the TP model transformation only the relevant $\Omega$ hypercube is considered from parameter space within $\mathbf{p}(t)$ changes during operation. From mathematical point of view this can be reached by convex hull manipulation. The recently developed Minimal Volume Simplex (MVS) convex hull [17, 37] provides the tightest range thus we applied this convex hull type in this study.

Compared to a "general" qLPV model, the main benefit of the TP kind qLPV model is that interprets the qLPV model only at given points in the $\Omega$ by introducing an $r$ sampling in $\Omega$ among all dimensions. In this way both the handled parameter space is more limited and the computational costs of the calculations is significantly lower regarding controller design and during operation. The TP kind qLPV model approximates the original qLPV model with given accuracy inside the predefined $\Omega$ hypercube [22]. The realization steps of the TP model transformation can be found in $[17,22,28,38]$. 
The state-feedback control uses the state variables or state variable estimates in the closed loop. In general the control signal can be calculated as follows in the continuous time domain if we consider an LPV kind state-feedback [20, 31]:

$$
\mathbf{u}(t)=-\mathbf{G}(\mathbf{p}(t)) \mathbf{x}(t)
$$

The $\mathbf{G}(\mathbf{p}(t)) \in \mathbb{R}^{m \times n}$ is the parameter dependent controller gain. The polytopic convex TP controller is the following (based on (22)):

$$
\mathbf{G}(\mathbf{p}(t))=\mathcal{G} \underset{\mathrm{r}=1}{\mathrm{R}} \mathbf{w}_{r}\left(p_{r}(t)\right)=\mathcal{G} \times{ }_{r} \mathbf{w}(\mathbf{p}(t)),
$$

where $\mathbf{G}(\mathbf{p}(t))$ is the parameter dependent feedback matrix, $\mathcal{G}$ is the feedback tensor consists of the $\mathbf{G}_{i_{1}, i_{2}, \ldots, i_{R}}$ feedback matrices belong to given $\mathbf{S}_{i_{1}, i_{2}, \ldots, i_{R}}$ LTI vertices and $\mathbf{w}_{r}\left(p_{r}(t)\right)$ is the convex weighting vector function (which is the same as in (20)). Further information regarding parameter dependent state-feedback can be found in [17, 31, 35, 39].

In the recent years the TP model transformation based modeling and control have been widely used in many fields e.g. industrial designs, physiological controls, physical modeling and control [40-59] thank to the continuous improvement of the method regarding computational relaxations $[60,61]$ and more effective convex hull manipulation [23, 28, 37, 62].

The $\mathbf{w}\left(\mathbf{p}_{r}\right)$ sampled weighting vector function obtained after the TP model transformation is applied on (19). The result is shown by Fig. 1 (the values are discrete ones, but the plotting function represent is as continuous). Naturally, the weighting vector function looks differently during operation while the $\mathbf{p}(t)$ is continuously varying which is caused the variation of $\mathbf{w}$ as well.

Remark 1 During the realization $\mathbf{w}(\mathbf{p}(t))$ should be applied due to the continuous controller. For that purpose we applied simple linear interpolation between values of $\mathbf{w}\left(\mathbf{p}_{r}\right)$ depends on the actual $\mathbf{p}(t)$.
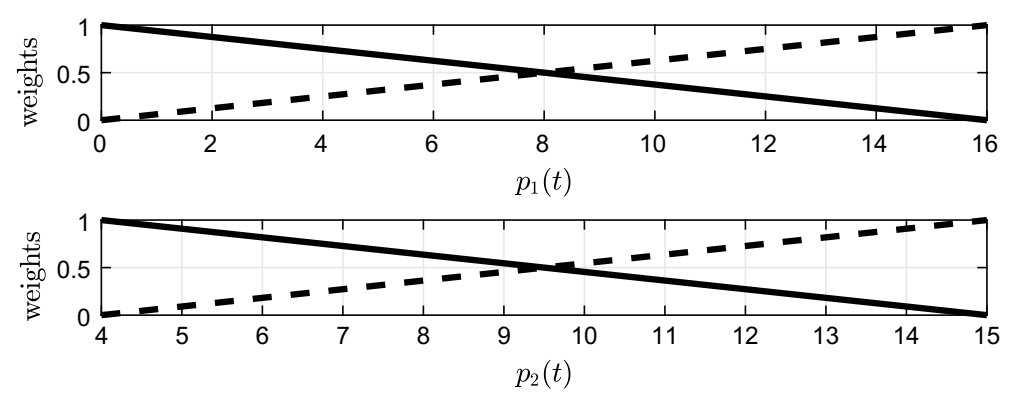

Fig. 1 The $\mathbf{w}\left(\mathbf{p}_{r}\right)$ applied in (20) and (23) equipped with the properties described in (21). [Upper subfigure: $\mathbf{w}_{1}\left(p_{1, r}\right)$; lower subfigure: $\left.\mathbf{w}_{2}\left(p_{2, r}\right)\right]$ 


\subsection{Linear Matrix Inequality Based Controller Design}

The basic formulation of a LMI is the following expression:

$$
\mathbf{F}(x):=\mathbf{F}_{0}+\sum_{i=1}^{m} \mathbf{x}_{i} \mathbf{F}_{i}>0
$$

where $\mathbf{x} \in \mathbb{R}^{m}, \mathbf{F}_{i}=\mathbf{F}_{i}^{\top} \in \mathbb{R}^{n \times n}$ and $i=1, \ldots, m$. In (24) the requirement against $\mathbf{F}(\mathbf{x})$ to be positive definite, namely, $\mathbf{z}^{\top} \mathbf{F}(\mathbf{x}) \mathbf{z}>0 \forall \mathbf{z} \in \mathbb{R}^{n}$. The inequality can be satisfied by using numerical optimization [63].

One direction of the possible LMI based controller design opportunity is based on the Lyapunov theorems. According to the Lyapunov theorem a given $\dot{\mathbf{x}}(t)=$ $\mathbf{A x}(t)$ system is stable if there can be constructed a positive quadratic $V(\mathbf{x})=\mathbf{x}^{\top} \mathbf{P} \mathbf{x}$ Lyapunov function which derivative is negative definite, namely $\dot{V}(\mathbf{x})=\mathbf{x}^{\top}\left(\mathbf{A}^{\top} \mathbf{P}+\right.$ $\mathbf{P A}) \mathbf{x}$. This criteria is satisfied if $\mathbf{A}^{\top} \mathbf{P}+\mathbf{P A}<\mathbf{0}$ and $\mathbf{P}=\mathbf{P}^{\top}>\mathbf{0}[22,64,65]$.

The criteria above can be applied in case of polytopic LPV systems given by their state space representation: $\dot{\mathbf{x}}(t)=\mathbf{A}(\mathbf{p}(t)) \mathbf{x}(t)+\mathbf{B}(\mathbf{p}(t)) \mathbf{u}(t)$ as in (10). Due to the polytopic representation $[\mathbf{A}(\mathbf{p}(t)) \mathbf{B}(\mathbf{p}(t))]=\sum_{r=1}^{R} w_{r}(\mathbf{p})\left[\mathbf{A}_{r} \mathbf{B}_{r}\right]$ [22] where $w_{r}(\mathbf{p})$ are the related convex weighting functions as (21). By taking into account the Lyapunov function candidate $V(\mathbf{x}(t))=\mathbf{x}^{\top} \mathbf{P} \mathbf{x}=\mathbf{x}^{\top} \mathbf{X}^{-1} \mathbf{x}$ a possible controller is the following [17]:

$$
\mathbf{u}(t)=\mathbf{M}(\mathbf{p}(t)) \mathbf{X}^{-1} \mathbf{x}(t)=\sum_{j=1}^{J} w_{j}(\mathbf{p}) \mathbf{M}_{j} \mathbf{X}^{-1} \mathbf{x}(t)
$$

At this point the $\dot{V}(\mathbf{x}(t))$ has to be investigated which can be described as follows after rearranging the Lyapunov function candidate's derivative:

$$
\dot{V}(\mathbf{x}(t))=\mathbf{x}^{\top}(t) \mathbf{X}^{-1} \cdot \operatorname{Sym}(\mathbf{A}(\mathbf{p}) \mathbf{X}+\mathbf{B}(\mathbf{p}) \mathbf{M}(\mathbf{p})) \cdot \mathbf{X}^{-1} \mathbf{x}^{\top}(t),
$$

where the "Sym" denotes symmetric matrix. The (26) can be written by using the polytopic weighting function description from above:

$$
\operatorname{Sym}(\mathbf{A}(\mathbf{p}) \mathbf{X}+\mathbf{B}(\mathbf{p}) \mathbf{M}(\mathbf{p}))=\sum_{i=1}^{R} \sum_{j=1}^{R} w_{i}(\mathbf{p}) w_{j}(\mathbf{p}) \operatorname{Sym}\left(\mathbf{A}_{i} \mathbf{X}+\mathbf{B}_{i} \mathbf{M}_{j}\right)<\mathbf{0} .
$$

Sophisticated control rules and requirements can be formalized by using LMI theorems. By solving the LMIs of the formalized convex objective function through numerical optimization obtaining controller candidate inherits the prescribed properties [66]. By involving the TP-qLPV model into the design steps the appearing TP controller will also be capable to act appropriately according to the predefined rules. 
During our investigations we have applied Parallel Distributed Compensation (PDC) kind control opportunity. PDC is a complex state-feedback kind controller based on quadratic stabilization [17]. Different LMI theorems can be involved into the impositions (e.g. pole clustering, $\mathrm{H}_{\infty}, \mathrm{H}_{2}$, etc.- - these rules are also applicable separately or simultaneously). In this examination we have employed the so-called LMI regions via pole clustering. Pole clustering allows the designer to aggregate the poles of the closed loop into a given domain in the complex plain-this domain is called as LMI region. Thus, by solving the given LMIs the closed loop poles will lie within this complex domain [30, 64].

Definition 1 A given $\mathcal{D}$ domain is an LMI region in the complex plane if $\exists \alpha$ that is $\alpha=\left[\alpha_{i j}\right] \in \mathbb{R}^{q \times q}$ and $\beta=\left[\beta_{i j}\right] \in \mathbb{R}^{c \times q}$ through which $\mathcal{D}:=\left\{z \in \mathbb{C}: f_{\mathcal{D}}(z)=\right.$ $\left.\alpha+\beta z+\beta^{\top} \bar{z}<0\right\}[65,67]$.

Definition 2 A $\dot{x}(t)=\mathbf{A} x(t)$ dynamical system is called as $\mathcal{D}$-stable if its poles lie in this $\mathcal{D}$ region (it is considered that the $\mathcal{D}$ region is in the negative half part of the complex plane) $[63,67]$.

Definition 3 The $\mathbf{A}$ is $\mathcal{D}$ stable if and only if $\exists \mathbf{X}>0$ symmetric positive definite matrix that is $M_{\mathcal{D}}(\mathbf{A}, \mathbf{X}):=\alpha \otimes \mathbf{X}+\beta \otimes \mathbf{A X}+(\beta \otimes \mathbf{A X})^{\top}<0$ in which $\otimes$ is the Kronecker-product [67].

The connection between the clustered poles, the properties of the state and Lyapunov matrix can be recognized in $f_{\mathcal{D}}(z)$ and $M_{\mathcal{D}}(\mathbf{A}, \mathbf{X})$ that is $(1, z, \bar{z}) \leftrightarrow$ $\left(\mathbf{X}, \mathbf{A X}, \mathbf{X} \mathbf{A}^{\top}\right)$ [67].

In consonance with [17, 21, 63, 64, 67] a suitably designed PDC kind controller with appropriate $\mathbf{G}(\mathbf{p}(t))$ gains (which follow the requirements of (22), (23), (25) and (27)) is able to satisfy the $\mathcal{D}$ stability requirements with respect to given system and enforces the poles of the closed loop to lie in the determined region of the complex plane and providing appropriate control action in case of additional requirements. By assuming that $\left.(\mathbf{A X}),(\mathbf{A X})^{\top} \leftrightarrow(\mathbf{A X}+\mathbf{B M}),(\mathbf{A X}+\mathbf{B M})^{\top}\right)$ during the control design an appropriate PDC controller may be obtained where $\mathbf{M}$ is the varying matrix (the subject of optimization) and the control gain can be calculated as $\mathbf{G}:=\mathbf{M X}^{-1}$.

In case of a polytopic qLPV system this assumption has to be extended to the vertices as it is described in [17, 22, 67]: $\left(\mathbf{A}_{\mathbf{i}} \mathbf{X}\right),\left(\mathbf{A}_{\mathbf{i}} \mathbf{X}\right)^{\top} \leftrightarrow\left(\mathbf{A}_{\mathbf{i}} \mathbf{X}+\mathbf{B} \mathbf{M}_{\mathbf{i}}\right),\left(\mathbf{A}_{\mathbf{i}} \mathbf{X}+\right.$ $\left.\mathbf{B} \mathbf{M}_{\mathbf{i}}\right)^{\top}$ ) where $\mathbf{M}_{i}$ is the varying matrix (the subject of optimization) and the control gains can be calculated by the $\mathbf{G}_{i}:=\mathbf{M}_{i} \mathbf{X}^{-1}$ equation. This is the same for TP-qLPV systems as well.

Remark 2 When we used this description that $\left(\mathbf{A}_{\mathbf{i}} \mathbf{X}\right),\left(\mathbf{A}_{\mathbf{i}} \mathbf{X}\right)^{\top} \leftrightarrow\left(\mathbf{A}_{\mathbf{i}} \mathbf{X}+\mathbf{B} \mathbf{M}_{\mathbf{i}}\right)$, $\left(\mathbf{A}_{\mathbf{i}} \mathbf{X}+\mathbf{B} \mathbf{M}_{\mathbf{i}}\right)^{\top}$ ) we considered that varying parameters can be found only in the $\mathbf{A}_{\mathbf{i}}$ state matrix. For complex summation rules related to the given LMI theorems if B is parameter dependent we make reference to [17, 64].

During our research we have considered two pole-clustering type LMIs, the $\alpha$ stability (28) and the disk region (29): 


$$
(\mathbf{X A}+\mathbf{B M})+(\mathbf{X A}+\mathbf{B M})^{\top}+2 \alpha \mathbf{X}<0
$$

and

$$
\left[\begin{array}{cc}
-r \mathbf{X} \\
-q \mathbf{X}+(\mathbf{X A}+\mathbf{B M})^{\top} & -q \mathbf{X}+(\mathbf{X A}+\mathbf{B M}) \\
-r \mathbf{X}
\end{array}\right]<0 .
$$

In (28) the $\alpha$ determines the boundary from which all of the closed loop poles lie towards negative direction while (29) determines a closed circle with $q$ center and $r$ radius into which the closed loop poles have to be fallen. By applying both LMI at the same time a half circle can be determined in which all of the closed loop poles will be found.

In order to describe the $\mathbf{G}_{i}$ gains in accordance with (23), the (28) and (29) have to be modified as follows by taking into account the details denoted above and in conformity with Remark 2.

$$
\begin{aligned}
& \text { Subjects : X, M } \\
& \mathbf{X}>0, \\
& \left(\mathbf{X} \mathbf{A}_{i}+\mathbf{B} \mathbf{M}_{i}\right)+\left(\mathbf{X} \mathbf{A}_{i}+\mathbf{B} \mathbf{M}_{i}\right)^{\top}+2 \alpha \mathbf{X}<0, \\
& \left(\mathbf{X} \mathbf{A}_{i}+\mathbf{B} \mathbf{M}_{j}\right)+\left(\mathbf{X} \mathbf{A}_{i}+\mathbf{B} \mathbf{M}_{j}\right)^{\top}+2 \alpha \mathbf{X}<0, \\
& {\left[\begin{array}{cc}
-r \mathbf{X} & -q \mathbf{X}+\left(\mathbf{X} \mathbf{A}_{i}+\mathbf{B} \mathbf{M}_{i}\right) \\
-q \mathbf{X}+\left(\mathbf{X} \mathbf{A}_{i}+\mathbf{B} \mathbf{M}_{i}\right)^{\top} & -r \mathbf{X}
\end{array}\right]<0,} \\
& {\left[\begin{array}{cc}
-r \mathbf{X} & -q \mathbf{X}+\left(\mathbf{X} \mathbf{A}_{i}+\mathbf{B} \mathbf{M}_{j}\right) \\
-q \mathbf{X}+\left(\mathbf{X} \mathbf{A}_{i}+\mathbf{B} \mathbf{M}_{j}\right)^{\top} & -r \mathbf{X}
\end{array}\right]<0,} \\
& i<j \leq R \text { s.t. } \forall \mathbf{p}(t): w_{i}(\mathbf{p}(t)) w_{j}(\mathbf{p}(t))=0,
\end{aligned}
$$

The representations of the applied LMIs can be found in Fig. 2. The Fig. 2c shows the case which has been applied in our examination, namely, the used parameters were the followings: $q, \alpha \leq 0$ and $r=12$. The selection of them have been arbitrary, but reasonable because in this domain the closed loop poles are stable, however, sufficiently "slow" to avoid high intervening (control) signals. By applying the mentioned LMIs in the represented way the closed loop poles fall into the given half circle and their stability is guaranteed if the LMIs are satisfied.

The $\mathbf{G}_{i}$ gains have obtained after solving (30). The LMIs represent a feasibility kind problem which can be solved by numerical iterative optimization. We applied the YALMIP core [68] and the MOSEK [69] solver in the MATLAB framework on (30) with respect to (19). The obtained $\mathbf{G}_{i}$ gains for the given vertices are the followings: $\mathbf{G}_{1}=\left[\begin{array}{ll}306.7595290 .6111 & -21.9195\end{array}\right], \mathbf{G}_{2}=[704.5794273 .4003-$ $21.2493], \mathbf{G}_{3}=[277.1904525 .6143-20.5256], \mathbf{G}_{4}=[646.2452524 .2035-$ 20.5060].

Remark 3 It should be noted that the calculation of the closed loop poles can be done as $\lambda\left(\mathbf{A}_{i}+\mathbf{B G}_{i}\right)$ due to the formulations in (25)-(27) and (30). Therefore, the negative sign in (22) must be positive during the application. 
(a)
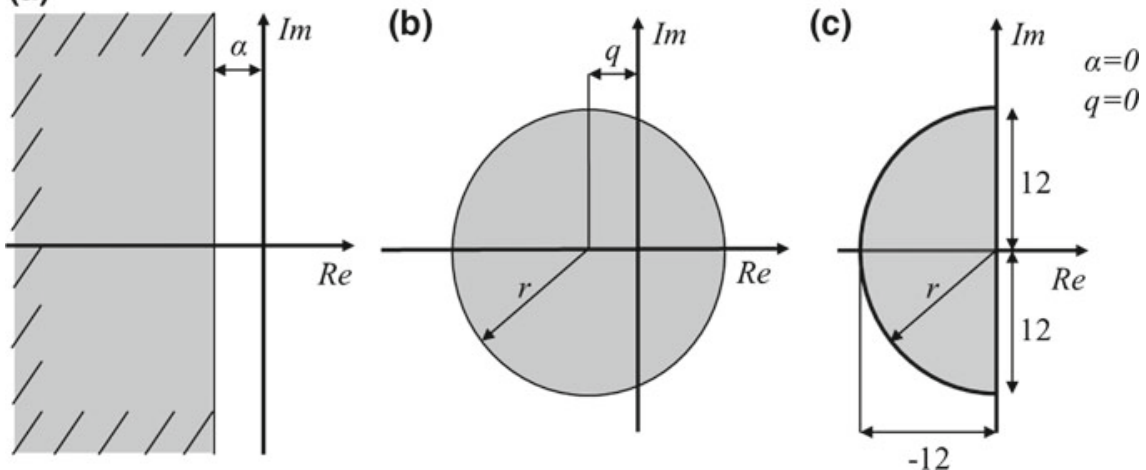

Fig. 2 Targeted $D$-region [a $\alpha$-stability in general, $\mathbf{b}$ disk region in general, $\mathbf{c}$ selected $D$-region]

Fig. $3 \lambda(\mathbf{A}+\mathbf{B G})$ poles of the closed system inside the $\mathcal{D}$ complex region. $[\mathrm{x}$ closed loop poles at vertices; * - closed loop poles during operation. Due to the overlapping poles in the plot the asterisks seem dots]

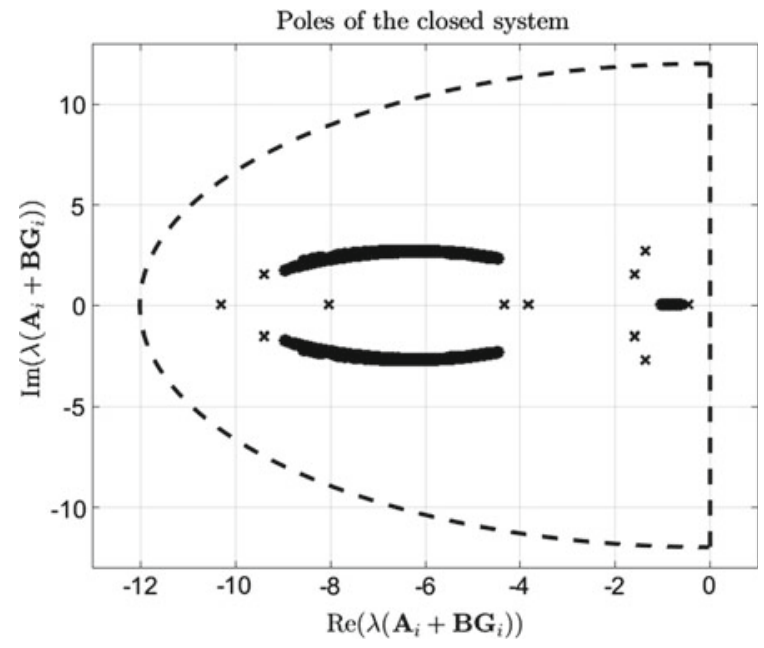

We have calculated the closed loop poles at the vertices which were: $\lambda\left(\mathbf{A}_{1}+\right.$ $\left.\mathbf{B G}_{1}\right)=[-0.6391-9.3912+1.5426 i-9.3912-1.5426 i]^{\top}, \lambda\left(\mathbf{A}_{2}+\mathbf{B G}_{2}\right)=$ $[-0.4528-10.2849-8.0136]^{\top}, \quad \lambda\left(\mathbf{A}_{3}+\mathbf{B G}_{3}\right)=[-1.3526+2.6762 i-$ $1.3526-2.6762 i-4.3225]^{\top}, \quad \lambda\left(\mathbf{A}_{4}+\mathbf{B G}_{4}\right)=[-1.582+1.5181 i-1.582-$ $1.5181 i-3.8442]^{\top}$.

The closed loop poles can be seen in Fig. 3 as well where we denoted the poles at the vertices by crosses and the poles which obtained during operation by asterisks. Despite the continuously varying resulting controller the closed loop poles lied within the determined LMI region. 


\subsection{Complementary Controller Design}

Due to the applied error dynamics based modeling the developed controller aims to cancel the deviation of the states of the model from given reference states and control signal. However, these reference states and control signal need to be "externally" provided. For that reason we have developed a complementary reference subsystem which includes a reference model and precompensator kind controller. The goal of this subsystem is to provide both the $\mathbf{x}_{r e f}(t)$ reference trajectories and $u_{r e f}(t)$ reference control signal need to be followed by the controlled nonlinear model. In order to design these signals we have applied the well-known inverse dynamics compensation completed by proportional-derivative compensator (IDC-PD) [70-72].

The connection between the control signal and controlled variable need to be determined in order to design an appropriate IDC-PD compensator as first step. According to the (4)-(6) the $u(t)$ control signal affects $\dddot{x}_{1}(t)$ (as a reminder, the $x_{1}(t)$ state variables is considered as measurable thus the control law has to be defined for that). For that purpose we have applied the previously developed reference model as described by (8). Therefore the mapping between the signals, namely, $\dddot{x}_{1}(t)$ should be elaborated by using (8):

$$
\begin{aligned}
& x_{1, \text { nom }}(t)=e^{(-\xi \cdot t)} \cdot x_{1, r e f}\left(t_{0}\right) \\
& \dot{x}_{1, \text { nom }}(t)=-\xi e^{(-\xi \cdot t)} \cdot x_{1, \text { ref }}\left(t_{0}\right) \\
& \ddot{x}_{1, \text { nom }}(t)=(-\xi)^{2} e^{(-\xi \cdot t)} \cdot x_{1, \text { ref }}\left(t_{0}\right) \\
& \dddot{x}_{1, \text { nom }}(t)=(-\xi)^{3} e^{(-\xi \cdot t)} \cdot x_{1, \text { ref }}\left(t_{0}\right)
\end{aligned}
$$

The general third order error compensator can be formulated as follows [71]:

$$
\begin{aligned}
& F \dddot{e}(t)+F_{1} \ddot{e}(t)+F_{2} \dot{e}(t)+F_{3} e(t)= \\
& F\left(\dddot{x}_{\text {nom }}(t)-\dddot{x}(t)\right)+F_{1}\left(\ddot{x}_{\text {nom }}(t)-\ddot{x}(t)\right)+F_{2}\left(\dot{x}_{\text {nom }}(t)-\dot{x}(t)\right) . \\
& +F_{3}\left(x_{\text {nom }}(t)-x(t)\right)=0
\end{aligned}
$$

In (32) $F, F_{1}, F_{2}$ and $F_{3}$ are the weighting parameters belong to the third, second, first and zero derivatives of the $e(t)=x_{\text {nom }}(t)-x(t)$ error function, respectively. Moreover, the $x_{n o m}(t)$ is the desired nominal state trajectory and $x(t)$ is the state variable need to be controlled.

A reference system can be an arbitrary system which describes the connection between the control signal and the controlled variable in a roughly approximate way-another key point is that the reference system need to be a third order one to fit both to the transformed model and the third order error compensator. In this study we have considered the transformed model (4)-(6) as reference systems as well and we assumed that both the model structure and parameters are known. In our later work we will investigate other reference systems as well to examine the robustness of the developed control framework. Therefore, the considered reference system was the following: 


$$
\begin{aligned}
& \dot{x}_{1, r e f}(t)=-\lambda_{1} x_{1, r e f}(t)+\lambda_{1} x_{2, r e f}(t) \\
& \dot{x}_{2, r e f}(t)=b e^{x_{1, r e f}(t)-x_{2, r e f}(t)}-d e^{2 x_{1, r e f}(t) / 3}-\eta x_{3, r e f}(t), \\
& \dot{x}_{3, r e f}(t)=-\lambda_{3} x_{3, r e f}(t)+u_{r e f}(t)
\end{aligned}
$$

The direct connection between $u_{r e f}(t)$ and $\dddot{x}_{1, r e f}(t)$ can be elaborated as follows by applying (33):

$$
\begin{aligned}
\dddot{x}_{1, r e f}(t) & =-\lambda_{1} \ddot{x}_{1, r e f}(t)+\lambda_{1} \ddot{x}_{2, r e f}(t) \\
& =-\lambda_{1} \ddot{x}_{1, r e f}(t)+\lambda_{1}\left(b e^{x_{1, r e f}(t)-x_{2, r e f}(t)}\left(\dot{x}_{1, r e f}(t)-\dot{x}_{2, r e f}(t)\right)\right. \\
& \left.-d \frac{2}{3} e^{2 x_{1, r e f}(t) / 3} \dot{x}_{1, r e f}(t)-\eta \dot{x}_{3, r e f}(t)\right) \\
& =-\lambda_{1} \ddot{x}_{1, r e f}(t)+\lambda_{1}\left(b e^{x_{1, r e f}(t)-x_{2, r e f}(t)} \dot{x}_{1, r e f}(t)-\dot{x}_{2, r e f}(t)\right. \\
& \left.-d \frac{2}{3} e^{2 x_{1, r e f}(t) / 3} \dot{x}_{1, r e f}(t)-\eta\left(-\lambda_{3} x_{3, r e f}(t)+u_{r e f}(t)\right)\right)
\end{aligned}
$$

We have assumed that $F=1 \mathrm{in}$ (32) which is a usual consideration in the literature $[71,72]$. The specific third order compensator can be realized by using (31), (32) and (34) in the following way:

$$
\begin{aligned}
& \dddot{e}(t)+F_{1} \ddot{e}(t)+F_{2} \dot{e}(t)+F_{3} e(t)=0 \\
& \left(\dddot{x}_{1, \text { nom }}(t)-\dddot{x}_{1, r e f}(t)\right)+F_{1}\left(\ddot{x}_{1, n o m}(t)-\ddot{x}_{1, r e f}(t)\right)+F_{2}\left(\dot{x}_{1, \text { nom }}(t)\right. \\
& \left.-\dot{x}_{1, \text { ref }}(t)\right)+F_{3}\left(x_{1, \text { nom }}(t)-x_{1, \text { ref }}(t)\right)=0 \\
& \left(\dddot{x}_{1, \text { nom }}(t)-\left(-\lambda_{1} \ddot{x}_{1, r e f}(t)+\lambda_{1}\left(b e^{x_{1, r e f}(t)-x_{2, r e f}(t)}\left(\dot{x}_{1, r e f}(t)-\dot{x}_{2, r e f}(t)\right) \cdot\right.\right.\right. \\
& \left.\left.\left.-d \frac{2}{3} e^{2 x_{1, r e f}(t) / 3} \dot{x}_{1, r e f}(t)-\eta\left(-\lambda_{3} x_{3, r e f}(t)+u_{r e f}(t)\right)\right)\right)\right)+ \\
& F_{1}\left(\ddot{x}_{1, \text { nom }}(t)-\ddot{x}_{1, \text { ref }}(t)\right)+F_{2}\left(\dot{x}_{1, n o m}(t)-\dot{x}_{1, \text { ref }}(t)\right)+ \\
& F_{3}\left(x_{1, \text { nom }}(t)-x_{1, \text { ref }}(t)\right)=0
\end{aligned}
$$

By rearranging (35) the $u_{\text {ref }}$ can be expressed as follows:

$$
\begin{aligned}
& L(t)=\dddot{x}_{1, \text { nom }}(t)-\lambda_{1} b e^{x_{1, r e f}(t)-x_{2, \text { ref }}(t)}\left(\dot{x}_{1, r e f}(t)-\dot{x}_{2, \text { ref }}(t)\right)+ \\
& \lambda_{1} d \frac{2}{3} e^{2 x_{1, r e f}(t) / 3} \dot{x}_{1, \text { ref }}(t)-\lambda_{1} \eta \lambda_{3} x_{3, r e f}(t)+F_{1}\left(\ddot{x}_{1, \text { nom }}(t)-\ddot{x}_{1, \text { ref }}(t)\right) \\
& +F_{2}\left(\dot{x}_{1, \text { nom }}(t)-\dot{x}_{1, \text { ref }}(t)\right)+F_{3}\left(x_{1, \text { nom }}(t)-x_{1, r e f}(t)\right) \\
& u_{r e f}(t)=\frac{L(t)}{-\lambda_{1} \eta}
\end{aligned}
$$

To summarize the operation, the application of $u_{r e f}(t)$ is the input for the (33) reference system, the $x_{1, \text { ref }}(t)$ behaves as the developed $x_{1, n o m}(t)$. In other words, the $u_{\text {ref }}(t)$ reference control signal assures that the $x_{1, \text { ref }}(t)$ smoothly follows $x_{1, n o m}(t)$ over time. 
Remark 4 During the calculation of $F_{1,2,3}$ it should be kept in mind that these parameters have to be positive and small scalers to be sure about the stability of the closed loop and the soft action of the IDC-PD compensator. The values can be calculated by determining the poles of (32) (in our case by determining the poles of (35)) characteristic equation. We applied the following poles: $\lambda_{1,2,3}=-0.05$. These poles provide the stability and soft control action as well for the closed loop with respect to the reference subsystem. By realizing the characteristics polynomial the $F_{1}=0.15, F_{2}=0.0075$, and $F_{3}=0.000125$ weights have been obtained.

\subsection{Extended Kalman Filter Design}

In order to finalize the control environment, the last missing piece is the state estimator which is needed due to the application of state-feedback kind PDC control. We decided to use a mixed continuous/discrete EKF since the physiological system to be controlled is continuous, however, the EKF usually uses discrete measurements (not mentioning the fact that it is realized on discrete systems) [25, 73, 74]. For the sake of simplicity we have considered the transformed Hahnfeldt model described by (4)-(6) during the EKF design. Naturally, this can be replaced with LPV based solution as well [75].

It should be noted that we have considered $T=1$ day sampling time which fits to the model properties.

The following functions describe the general mixed continuous/discrete EKF [25]:

$$
\begin{aligned}
& \dot{\mathbf{x}}(t)=f(\mathbf{x}(t), \mathbf{u}(t))+\mathbf{d}(t), \quad \mathbf{d}(t) \sim \mathcal{N}(\mathbf{0}, \mathbf{Q}) \\
& \mathbf{y}_{k}=h\left(\mathbf{x}_{k}\right)+\mathbf{v}_{k}, \quad \mathbf{v}_{k} \sim \mathcal{N}\left(\mathbf{0}, \mathbf{R}_{k}\right)
\end{aligned}
$$

In (37) the $f(\mathbf{x}(t), \mathbf{u}(t))$ is the equation of the system and $h\left(\mathbf{x}_{k}\right)$ is the sensor model while $\mathbf{x}_{k}=\mathbf{x}\left(t_{k}\right)$. Disturbances and noise have been also considered: the $\mathbf{d}(t)$ and $\mathbf{v}_{k}$ are the continuous disturbance signal and $\mathbf{v}_{k}$ is the discrete sensor noise signal, respectively.

Remark 5 The $f(\mathbf{x}(t), \mathbf{u}(t))$ is equal to (4)-(6). We have also considered that $h\left(\mathbf{x}_{k}\right)$ sensor model is equal to $x_{1}(t)$-which is the only measurable state variable. In our future work we will examine the opportunity the incorporation of more advanced sensor model.

With respect to the model properties the considered $\mathbf{d}(t)$ disturbance vector consists of normally distributed random variables with zero mean and 0.05 standard distribution. Hence, $\mathbf{Q}=\operatorname{cov}\left(\operatorname{diag}\left[0.05^{2}, 0.05^{2}, 0.05^{2}\right]\right)$. We assumed $\mathbf{R}_{k}=0.01^{2}$ due to the only one measurable output we have is the $x_{1}(t)$ state variable. The considered initial conditions have been the followings: $\hat{\mathbf{x}}_{0 \mid 0}=\mathrm{E}\left[\mathbf{x}\left(t_{0}\right)\right]$ estimated initial state vector and $\mathbf{P}_{0 \mid 0}=\operatorname{Var}\left[\mathbf{x}\left(t_{0}\right)\right]$ error covariance matrix. 


$$
\begin{aligned}
\dot{\hat{\mathbf{x}}}(t) & =f(\hat{\mathbf{x}}(t), \mathbf{u}(t)) \\
\dot{\mathbf{P}}(t) & =\mathbf{F}(t) \mathbf{P}(t)+\mathbf{P}(t) \mathbf{F}^{\top}(t)+\mathbf{Q},
\end{aligned} .
$$

In $\hat{\mathbf{x}}\left(t_{k-1}\right)=\hat{\mathbf{x}}_{k-1 \mid k-1}, \mathbf{P}\left(t_{k-1}\right)=\mathbf{P}_{k-1 \mid k-1}$ and $\mathbf{F}(t)=\left.\frac{\partial f}{\partial \mathbf{x}}\right|_{\hat{\mathbf{x}}, \mathbf{u}}$.

In the update phase these results have been applied as $\hat{\mathbf{x}}_{k \mid k-1}=\hat{\mathbf{x}}\left(t_{k}\right)$ and $\mathbf{P}_{k \mid k-1}=$ $\mathbf{P}\left(t_{k}\right)$.

Based on the previous findings, the Kalman gain can be calculated as

$$
\mathbf{K}_{k}=\mathbf{P}_{k \mid k-1} \mathbf{H}_{k}^{\top}\left(\mathbf{H}_{k} \mathbf{P}_{k \mid k-1} \mathbf{H}_{k}^{\top}+\mathbf{R}_{k}\right)^{-1}
$$

where $\mathbf{H}_{k}=\left.\frac{\partial h}{\partial \mathbf{x}}\right|_{\hat{\mathbf{x}}_{k \mid k-1}}$ which is in this particular case $\mathbf{H}_{k}=[1,0,0]$.

During the EKF design the last step is the update phase in which the predictions are corrected concerning to the measurements by using the calculated Kalman gain where $\mathbf{I}$ is the identity matrix:

$$
\begin{aligned}
& \hat{\mathbf{x}}_{k \mid k}=\hat{\mathbf{x}}_{k \mid k-1}+\mathbf{K}_{k}\left(\mathbf{y}_{k}-h\left(\hat{\mathbf{x}}_{k \mid k-1}\right)\right) \\
& \mathbf{P}_{k \mid k}=\left(\mathbf{I}-\mathbf{K}_{k} \mathbf{H}_{k}\right) \mathbf{P}_{k \mid k-1}
\end{aligned} .
$$

\subsection{Finalized Control Scheme}

The finalized control scheme can be seen in Fig. 4. It consists of the reference subsystem and the control framework.

The reference subsystem is an important, but replaceable part of the scheme. It is responsible to generate the $\mathbf{x}_{r e f}(t)$ reference state trajectories and the $u_{r e f}(t)$ reference control signal which are utilized by the control framework during the realization of the error based dynamical descriptions (namely to realize the $\Delta \hat{\mathbf{x}}_{r e f}(t)$ difference based estimated state variables and $u(t)$ applied control signal). The development of the belonging parts of the reference subsystem was described in Sects. 2.3 and 4.3.

The TP-LPV-LMI controller presented by Fig. 4 is based on the findings of Sects. 4.1-4.2 in a form of (22), while the EKF is based on Sect.4.4.

The considered nonlinear model in the control framework was the model described by (4)-(6).

The applied deviation based state reference signal is $\Delta \mathbf{r}=\mathbf{0}$ which means that the goal of the state feedback kind control is $\mathbf{x}(t)=\mathbf{x}_{r e f}(t)$ while $t \rightarrow \infty$. Thus, the control framework enforces the $\mathbf{x}_{r e f}=\mathbf{x}$ equality over time. 


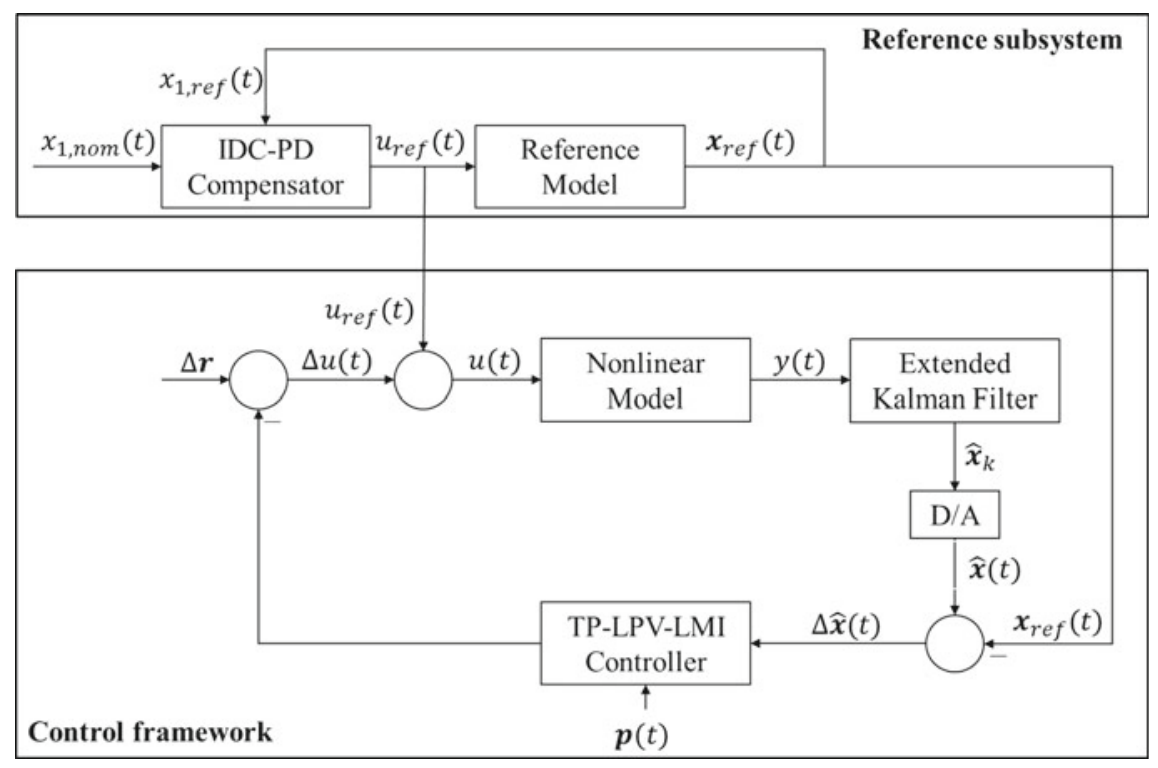

Fig. 4 Finalized control scheme

Finally, the generation of $\mathbf{p}(t)$ is the last missing piece. In (17)-(18) the $\mathbf{x}(t)$ state variables are needed in the realization of the parameter vector. However, $\mathbf{x}_{2,3}(t)$ are not available directly and $\mathbf{x}_{1}(t)$ is also loaded with noise. In the practical realization this issue can be solved by using the $\hat{\mathbf{x}}(t)$ estimated state variables instead of $\mathbf{x}(t)$. In this way, the (17)-(18) need to be modified as follows by considering the $\mathbf{x}_{r e f}(t)$ is known as it was described previously:

$$
\begin{gathered}
p_{1}(t)=\left\{\begin{array}{cl}
b e^{-\hat{x}_{2}(t)} \frac{\left(e^{\hat{x}_{1}(t)}-e^{x_{1, r e f}(t)}\right)}{\Delta \hat{x}_{1}(t)} & \text { if } \Delta \hat{x}_{1}(t) \neq 0 \\
-d \frac{\left(e^{2 \hat{x}_{1}(t) / 3}-e^{2 x_{1, r e f}(t) / 3}\right)}{\Delta \hat{x}_{1}(t)}, & \text { if } \Delta \hat{x}_{1}(t)=0 \\
\left(e^{\hat{x}_{1}(t)}+e^{x_{1, r e f}(t)}\right)\left(\frac{2}{3} d+b e^{2 \hat{x}_{2}(t)}\right), & \text { if } \Delta \hat{x}_{2}(t) \neq 0
\end{array}\right. \\
p_{2}(t)= \begin{cases}b e^{-x_{1, r e f}(t)} \frac{\left(e^{\hat{x}_{2}(t)}-e^{x_{2, r e f}(t)}\right)}{\Delta \hat{x}_{2}(t)}, & \text { if } \Delta \hat{x}_{2}(t)=0 \\
-b e^{x_{1, r e f}(t)}\left(e^{\hat{x}_{2}(t)}+e^{x_{2, r e f}(t)}\right),\end{cases}
\end{gathered}
$$




\section{Results and Discussion}

The simulation environment consists of two parts. The initialization, modeling and control design have been done in the MATLAB core. The numerical simulations have run in SIMULINK environment, however.

The circumstances considered during the numerical simulations were the following. The numerical values are arbitrarily selected, however, in accordance with our previous findings $[6,16]$. Due to $x_{1}(t)$ is the measurable output we assumed that $x_{1}\left(t_{0}\right)=\ln (14900)=9.6091$ is known. We selected that $x_{2}\left(t_{0}\right)=x_{1}\left(t_{0}\right)$ in conjunction to our previous studies $[6,16]$. The $x_{3}\left(t_{0}\right)=0$, namely, there was no drug administration before the start of the therapy. The initial state variables of the EKF have been selected alongside the following assumptions. The $\hat{x}_{1}\left(t_{0}\right)=x_{1}\left(t_{0}\right)=$ $\ln (14900)=9.6091$ since $x_{1}(t)$ is measurable and $\hat{x}_{3}\left(t_{0}\right)=0$ similarly to $x_{3}\left(t_{0}\right)=0$. The $\hat{x}_{2}\left(t_{0}\right)=\ln (18159)=9.8069 \mathrm{~mm}^{3}$ considered as slightly perturbated and different than $x_{2}\left(t_{0}\right)$. The $\mathbf{x}_{\text {ref }}\left(t_{0}\right)=[\ln (17000), \ln (17000), 0]^{\top}=[9.7410,9.7410,0]^{\top}$.

The final numerical values of the state variables after the end of the simulation have been the following:

- $\mathbf{x}\left(t_{\text {final }}\right)=[\ln (1.0245), \ln (1.0552), 8.9604]^{\top}=[0.0242,0.0537,8.9604]^{\top}$

- $\hat{\mathbf{x}}\left(t_{\text {final }}\right)=[\ln (1.0334), \ln (1.1363), 8.7441]^{\top}=[0.0329,0.1278,8.7441]^{\top}$;

- $\mathbf{x}_{\text {ref }}\left(t_{\text {final }}\right)=[\ln (1.054), \ln (1.0451), 8.929]^{\top}=[0.0526,0.0441,8.929]^{\top}$.

Figure 5 shows the decay of state variables over time. The upper sub-figure represents the original model $\mathbf{x}(t)$ to be controlled, the middle sub-figure is the state variables of the EKF $\hat{\mathbf{x}}(t)$, while the lower sub-figure belongs to the reference model $\mathbf{x}_{r e f}(t)$. The disturbances and noise can be observed on $\mathbf{x}(t)$ which reflect in the $\hat{\mathbf{x}}(t)$ as well. As one can see the final values of state variables of the model to be controlled (detailed above) reached the close environment of the determined therapeutic goalhowever, because of the disturbances and noises a small deviation obtained. The other aim of the controller is to enforce $\mathbf{x}(t)$ to behave as $\mathbf{x}_{r e f}(t)-$ which requirement has also been satisfied.

This latter conclusion can be strengthen qualitatively and quantitatively by investigating Fig. 6 and the root mean square error (RMSE) values, respectively. The RMSE values consider the difference between the models and state variables under the whole simulation horizon. The following list presents the RMSE values.

(a) $\operatorname{RMSE}_{\mathbf{x}(t)-\hat{\mathbf{x}}(t)}=[0.1033,0.2357,0.4422]$;

(b) $\operatorname{RMSE}_{\mathbf{x}(t)-\mathbf{x}_{r e f}(t)}=[0.023,0.0091,0.2944]$;

(c) $\operatorname{RMSE}_{\mathbf{x}_{r e f}(t)-\hat{\mathbf{x}}(t)}=[0.1041,0.2364,0.398]$.

The numerical RMSE results correspond to the expectations. The highest differences obtained when we compared the EKF's state variables $\hat{\mathbf{x}}(t)$ to the original and reference models (a and $\mathbf{c}$ cases). The performance of the control environment can be judged based on the $\mathbf{b}$ case as well. Despite the applied disturbances and noises the model to be controlled followed the reference model with low error. The control's aim was determined as $\Delta x_{1,2,3}(t)=0$ over $t \rightarrow 200$ (where 200 was the final day of 

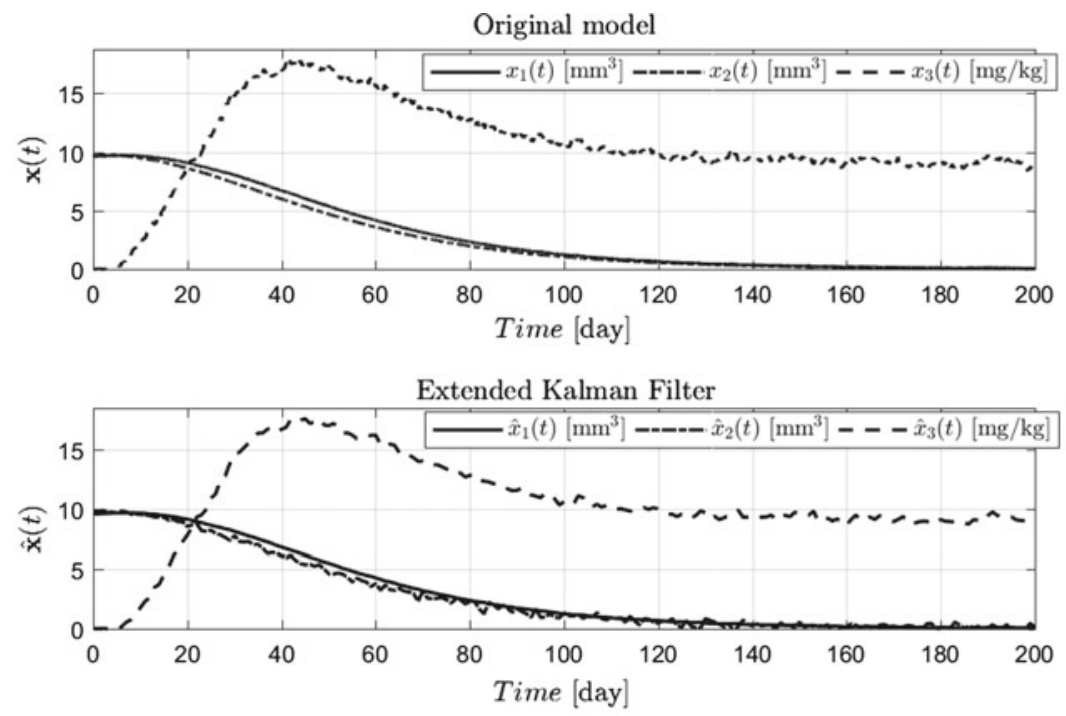

Reference model

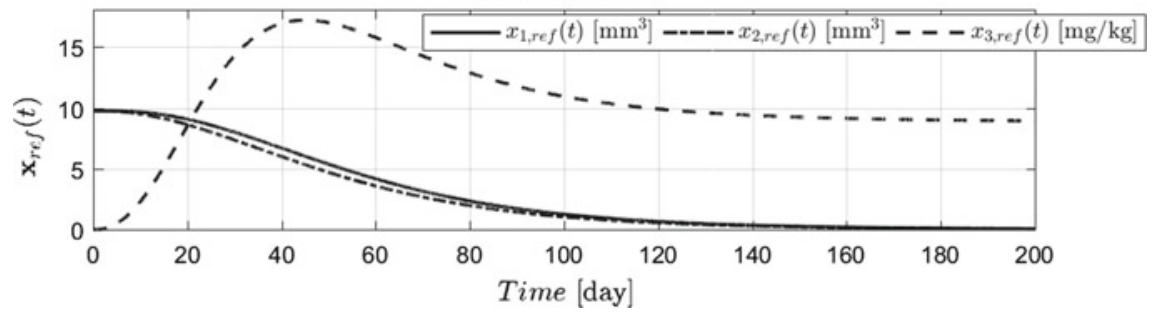

Fig. 5 Trajectories of state variables during operation

simulated therapy). According to the $\mathbf{x}\left(t_{\text {final }}\right)$ the controller environment was able to satisfy this requirement (Fig. 6). The main source of differences between the state variables of systems caused by the effect of disturbances and noise, however, the magnitudes of the deviations were approximately 0.3 in case of $\|\mathbf{x}(t)-\hat{\mathbf{x}}(t)\|$ and $\left\|\mathbf{x}_{r e f}(t)-\hat{\mathbf{x}}(t)\right\|$ and 0.15 in case of $\left\|\mathbf{x}_{r e f}(t)-\mathbf{x}(t)\right\|$ which is an acceptably low value with respect to $\mathbf{d}(t)$ and $\mathbf{v}_{k}$.

The applied transformed model originated from the described Hahnfeldt modelthus, it is meaningful to investigate the control effects on the Hahnfeldt model as well. For that purpose a reverse transformation $\left(z_{1,2}=\exp \left(x_{1,2}\right)\right)$ of the first two states can be done. The third state variable are identical, namely, $z_{3}=x_{3}$. The result can be seen in Fig. 7. It is clearly visible that the control framework performed well and it decreased the tumor and vasculature volumes in the predefined way.

Figure 8 shows the applied control signals. The upper subfigure is the $u_{\text {ref }}(t)$ reference control signal provided by the reference subsystem which guarantees the appropriate values of $\mathbf{x}_{r e f}(t)$ over time. As it can be seen in the middle subfigure the $u(t)$ provided by the control framework was quite similar to $u_{r e f}(t)$. The main 

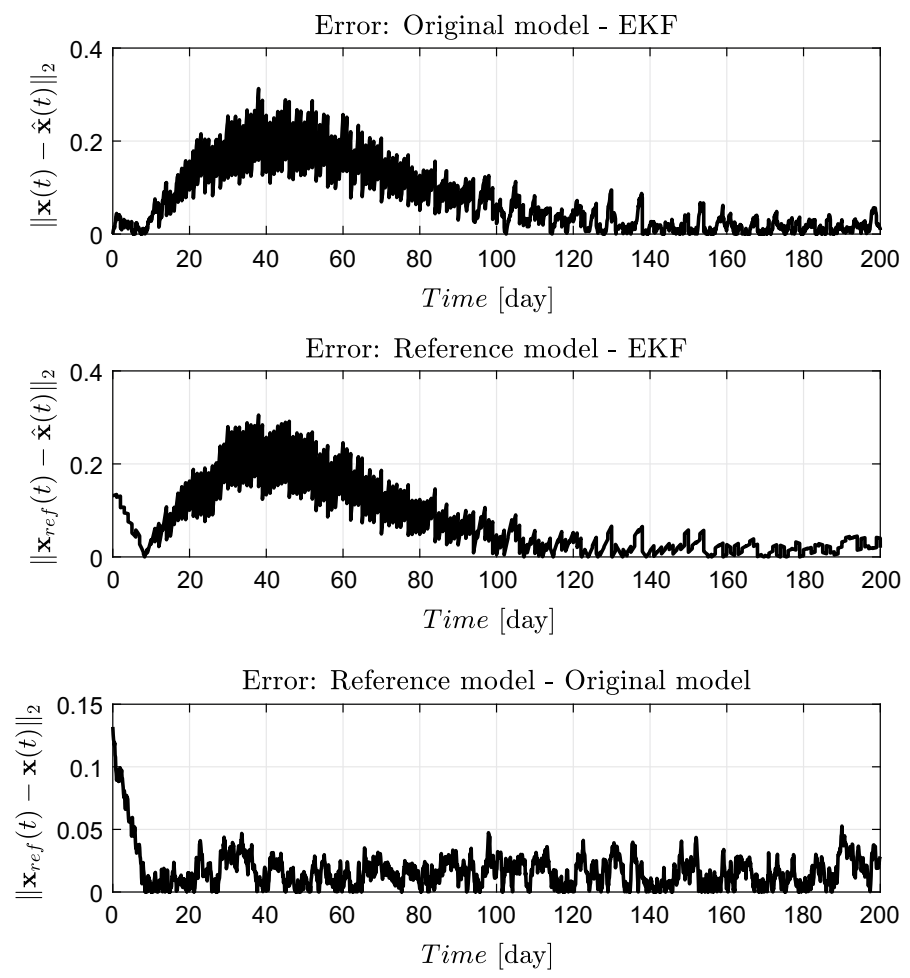

Fig. 6 2-norm based error representations between the state variables of the systems

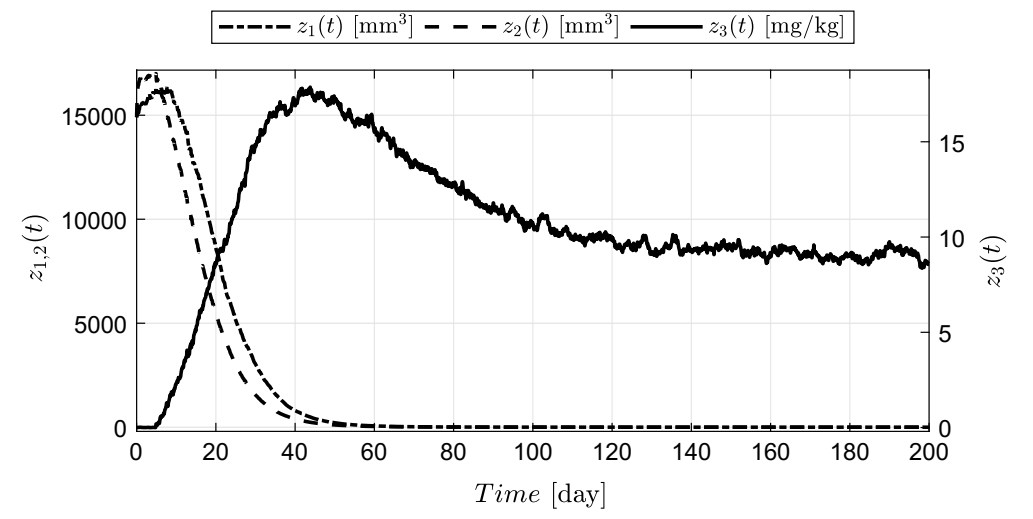

Fig. 7 State variables of the controlled model after reverse transformation 

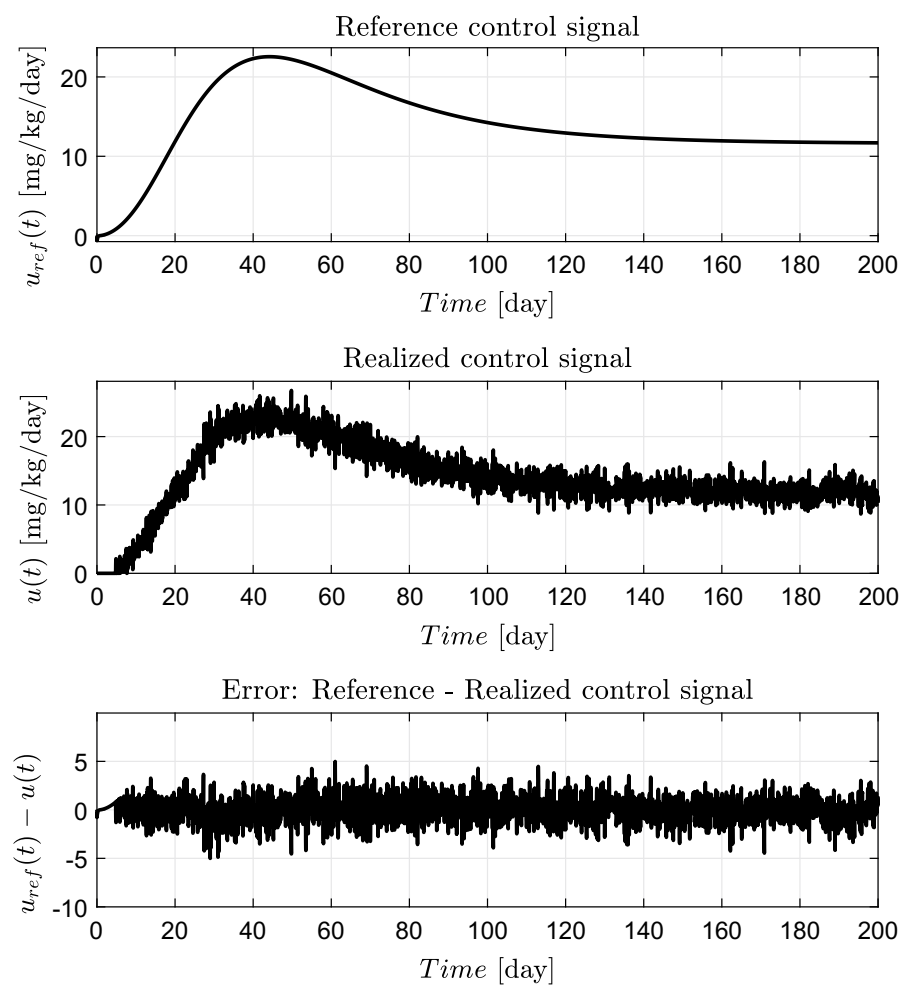

Fig. 8 Obtained control signals

source of difference (lower sugfigure) was that $u(t)$ was loaded by the effect of disturbances and noise due to signal smoothers have not been applied on $\hat{\mathbf{x}}(t)$ (this is also corroborated by Fig. 9 as well). The application of $\hat{\mathbf{x}}(t)$ affected the generated $u(t)$ from two sides. The first one that according to (22)-(23) the state feedback kind control law need to be applied as $\mathbf{u}(t)=-\mathbf{G}(\mathbf{p}(t)) \hat{\mathbf{x}}(t)$. The second effect is coming from $\mathbf{G}(\mathbf{p}(t))=\mathcal{G} \times_{r} \mathbf{w}(\mathbf{p}(t))$ in which the $\mathbf{p}(t)$ is calculated as (41)-(42), namely, different terms of $\hat{\mathbf{x}}(t)$ have been applied here as well. The consequence is the "noisy" resulting $u(t)$ control signal. However, by applying smoothers as the part of the EKF this effect can be minimized. This will be investigated in our future work.

Figure 9 shows the integrated control signals and their difference. In the upper subfigure the integrated time signals are almost totally aliasing-which corroborates that the main difference between $u(t)$ and $u_{\text {ref }}(t)$ is coming from the disturbances and noise effects. The lower subfigure shows the difference between them and indicated that the $u(t)$ and $u_{r e f}(t)$ is quite similar to each other. An important marker is the total injected amount of drug. In this case these values have been $U_{r e f}=2807.5[\mathrm{mg} / \mathrm{kg}]$ and $U=2798.2[\mathrm{mg} / \mathrm{kg}]$ in the simulated time horizon-which also strengthen that the discrepancy was minimal between the signals during operation. 

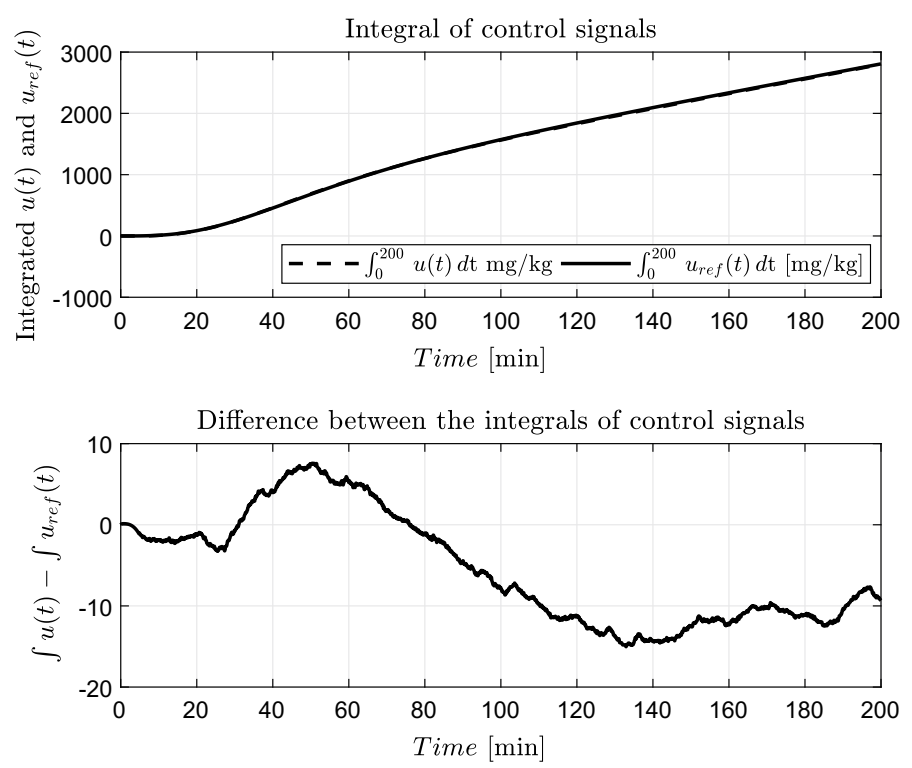

Fig. 9 Integrated control signals

Fig. 10 Vary of $\mathbf{p}(t)$ during operation

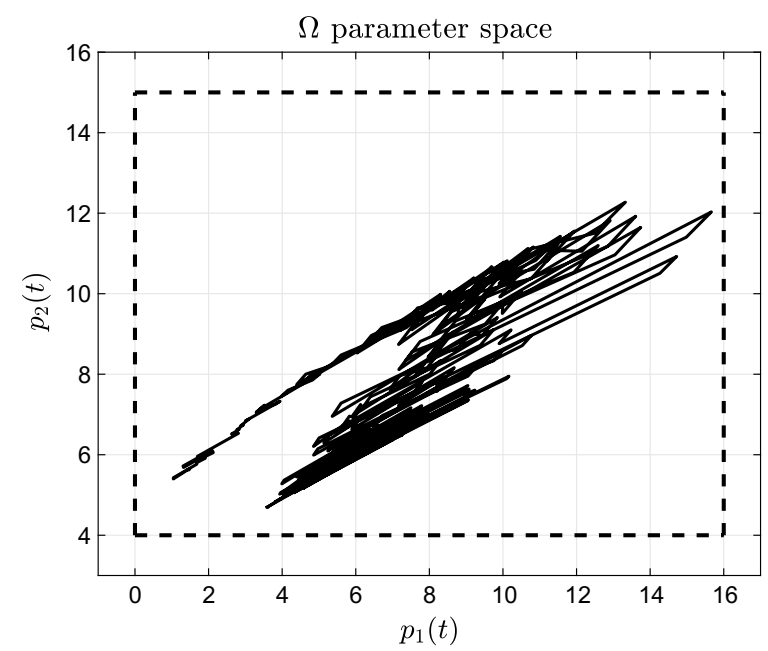



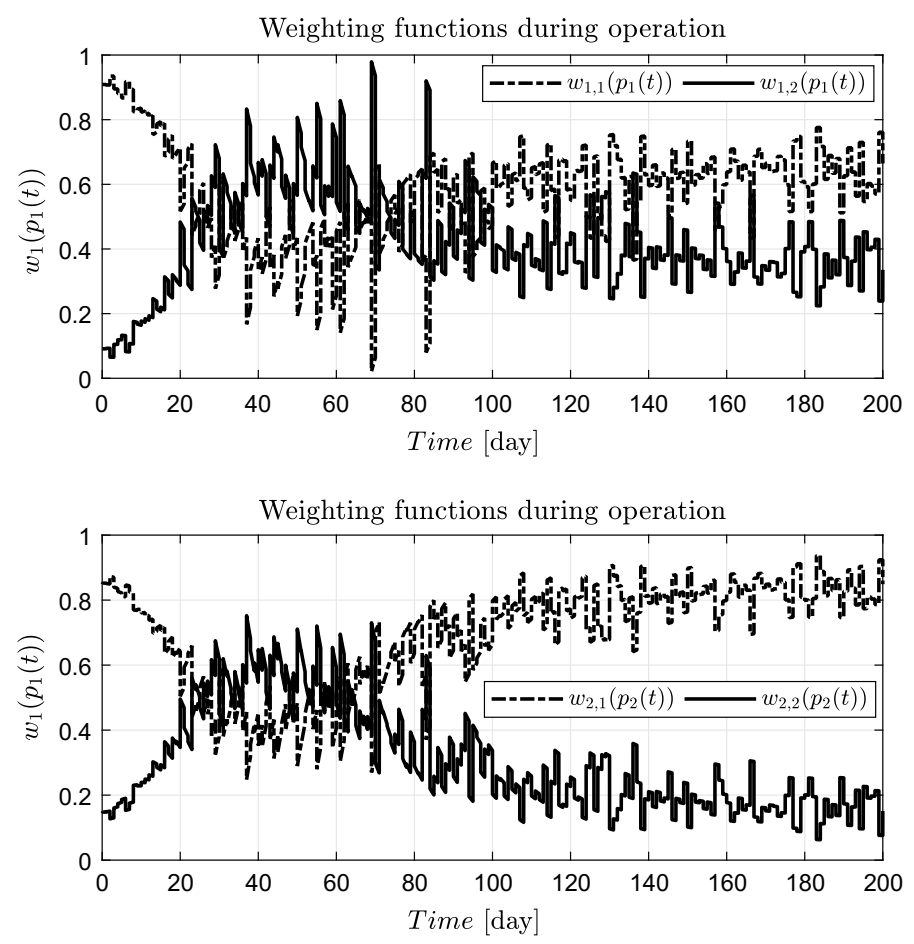

Fig. 11 Very of convex weighting functions during operation

An important indicator of the control framework is the behavior of the closed loop poles, $\mathbf{p}(t)$ and $\mathbf{w}(\mathbf{p}(t))$, respectively. Regarding the closed loop poles we refer to Fig. 2 on which the obtained closed loop poles were shown. The related details are described in Sect. 4.2. In order to check the domain violations the values of $\mathbf{p}(t)$ need to be investigated. Figure 10 shows the values of $\mathbf{p}(t)$ during operation in which the $\Omega$ domain of scheduling parameters are denoted by dashed lines. It can be seen that there is no domain violation. The other important indicator is the behavior of $\mathbf{w}(\mathbf{p}(t))$ as it is operated which can be seen in Fig. 11. The control framework performed well from this point of view as well and the (21) is satisfied during operation.

\section{Conclusion}

The study describes our latest results concerning tumor growth control by applying anti-angiogen therapy. We have applied a transformed model of Hahnfeldt and from it a specific qLPV model have developed on which the control design have been executed. A reference subsystem was also introduced to describe the behavior of the first state and this has been applied to generate reference trajectory. 
We detailed the control design as a step-by-step procedure. First, the control oriented qLPV model was converted to a TP-qLPV model trough TP model transformation. The control goals were determined with respect to the final values of the state variables to be controlled. These goals have been embedded into the LMIs. In this way the obtained resulting controller was ready to act according to them and finally to satisfy them.

The developed control framework has been examined by using numerical simulations. According to the results all of the control goals have reached-and the tumor and vasculature volumes decreased as prescribed.

In our future work we will investigate more complex model with the same controller design procedure, moreover, we examine the use of smoothers at the EKF side.

\section{Appendix}

\section{Notations and Abbreviations}

For the notations and abbreviations applied in this study please see Tables 1 and 2.

Table 1 General Phrases

\begin{tabular}{l|l}
\hline Abbreviation & Meaning \\
\hline LTI & Linear time invariant \\
\hline LTV & Linear time variant \\
\hline LPV & Linear parameter varying \\
\hline qLPV & quasi LPV \\
\hline TP model & Tensor product model \\
\hline LMI & Linear matrix inequality \\
\hline MVS & Minimal volume simplex \\
\hline SVD & $\begin{array}{l}\text { Singular value } \\
\text { decomposition }\end{array}$ \\
\hline HOSVD & Higher-order SVD \\
\hline EKF & Extended kalman filter \\
\hline
\end{tabular}


Table 2 Mathematical terms

\begin{tabular}{l|l}
\hline Notation & Meaning \\
\hline$a, b, \ldots$ & Scalars \\
\hline $\mathbf{a}, \mathbf{b}, \ldots$ & Vector \\
\hline $\mathbf{A}, \mathbf{B}, \ldots$ & Matrices \\
\hline $\mathbf{a}_{i}, \mathbf{b}_{i}, \ldots$ & $\begin{array}{l}\text { ith row vector of } \mathbf{A}, \mathbf{B}, \ldots \\
\text { matrices }\end{array}$ \\
\hline$a_{i, j}, b_{i, j}, \ldots$ & $\begin{array}{l}\text { jth elements of the } \mathbf{a}_{i}, \mathbf{b}_{i}, \ldots \\
\text { row vectors }\end{array}$ \\
\hline $\mathcal{A}, \mathcal{B}, \ldots$ & Tensors \\
\hline $\mathcal{S} \underset{\mathrm{n}=1}{\mathrm{~N}} \mathbf{W}_{n}$ & $\begin{array}{l}\text { Multiple tensor products, e.g. } \\
\mathcal{S} \times \mathbf{W}_{1} \ldots \times \mathbf{W}_{N} \mathbf{W}_{N}\end{array}$ \\
\hline $\mathbb{R}, \mathbb{C}, \ldots$ & Mathematical sets \\
\hline
\end{tabular}

\section{References}

1. K. Xing, S. Lisong, Molecular targeted therapy of cancer: the progress and future prospect. Front. Lab. Med. 1(2), 69-75 (2017)

2. P. Charlton, J. Spicer, Targeted therapy in cancer. Medicine 44(1), 34-38 (2016)

3. N.S. Vasudev, A.R. Reynolds, Anti-angiogenic therapy for cancer: current progress, unresolved questions and future directions. Angiogenesis 17(3), 471-494 (2014)

4. A.M.E. Abdalla, L. Xiao, M.W. Ullah, M. Yu, C. Ouyang, G. Yang, Current challenges of cancer anti-angiogenic therapy and the promise of nanotherapeutics. Theranostics $8(2), 533-$ $548(2018)$

5. Y. Kubota, Tumor angiogenesis and antiangiogenic therapy. Keio J. Med. 61, 47-56 (2012)

6. J. Sápi, Controller-managed automated therapy and tumor growth model identification in the case of antiangiogenic therapy for most effective, individualized treatment. Ph.D. Thesis, Óbuda University, Budapest, Hungary, 2015

7. C. Ionescu, R. De Keyser, J. Sabatier, A. Oustaloup, F. Levron, Low frequency constant-phase behavior in the respiratory impedance. Biomed. Signal Process. 6(2), 197-208 (2011)

8. D. Copot, R. De Keyser, J. Juchem, C.M. Ionescu, Fractional order impedance model to estimate glucose concentration: in vitro analysis. ACTA Polytech. Hung. 14(1), 207-220 (2017)

9. L. Kovács, A robust fixed point transformation-based approach for type 1 diabetes control. Nonlinear Dyn. 89(4), 2481-2493 (2017)

10. C. Ionescu, A. Lopes, D. Copot, J.A.T. Machado, J.H.T. Bates, The role of fractional calculus in modeling biological phenomena: a review. Commun. Nonlinear Sci. 51, 141-159 (2017)

11. A. Dineva, J.K. Tar, A. Várkonyi-Kóczy, V. Piuri, Adaptive controller using fixed point transformation for regulating propofol administration through wavelet-based anesthetic value, in 2016 IEEE International Symposium on Medical Measurements and Applications (MeMeA) (IEEE, 2016), pp. 1-6

12. F.S. Lobato, V.S. Machado, V. Steffen, Determination of an optimal control strategy for drug administration in tumor treatment using multi-objective optimization differential evolution. Comput. Methods Programs Biomed. 131, 51-61 (2016)

13. D. Drexler, J. Sápi, L. Kovács, Potential benefits of discrete-time controller-based treatments over protocol-based cancer therapies. Acta Polytech. Hung. 14(1), 11-23 (2017)

14. J. Klamka, H. Maurer, A. Swierniak, Local controllability and optimal control for a model of combined anticancer therapy with control delays. Math. Biosci. Eng. 14(1), 195-216 (2017) 
15. D.A. Drexler, J. Sápi, L. Kovács, Modeling of tumor growth incorporating the effects of necrosis and the effect of bevacizumab. Complexity 2017, 1-10 (2017)

16. D. Drexler, J. Sápi, L. Kovács, Positive nonlinear control of tumor growth using angiogenic inhibition. IFAC-PapersOnLine 50(1), 15068-15073 (2017). (20th IFAC World Congress)

17. J. Kuti, Generalization of tensor product model based control analysis and synthesis. Ph.D. Thesis, Applied Informatics and Applied Mathemathics Doctoral School, Óbuda University, Budapest, Hungary, 2018

18. P.H. Colmegna, R.S. Sanchez-Pena, R. Gondhalekar, E. Dassau, F.J. Doyle, Switched LPV glucose control in type 1 diabetes. IEEE Trans. Biomed. Eng. 63(6), 1192-1200 (2016)

19. L. Kovács, Linear parameter varying (LPV) based robust control of type-I diabetes driven for real patient data. Knowl-Based Syst. 122, 199-213 (2017)

20. S. Boyd, L. El Ghaoui, E. Feron, V. Balakrishnan, Linear Matrix Inequalities in System and Control Theory, vol. 15 (Siam, 1994)

21. G. Herrmann, M.C. Turner, I. Postlethwaite, Linear matrix inequalities in control, in Mathematical Methods for Robust and Nonlinear Control (Springer, Berlin, 2007), pp. 123-142

22. P. Baranyi, Y. Yam, P. Varlaki, Tensor Product Model Transformation in Polytopic Model-Based Control, 1 st edn. (CRC Press, USA, 2013)

23. A. Szollosi, P. Baranyi, Influence of the tensor product model representation of qLPV models on the feasibility of linear matrix inequality based stability analysis. Asian J. Control 20(1), 531-547 (2018)

24. Gy. Eigner, L. Kovács, Linear matrix inequality based control of tumor growth, in 2017 IEEE International Conference on Systems, Man and Cybernetics, ed. by L. Deng (IEEE Systems, Man and Cybernetics Society, New York, 2017), pp. 1734-1739

25. M.S. Grewal, A.P. Andrews, Kalman Filtering: Theory and Practice Using MATLAB, 3rd edn. (Wiley, Chichester, 2008)

26. P. Hahnfeldt, D. Panigrahy, J. Folkman, L. Hlatky, Tumor development under angiogenic signaling: a dynamical theory of tumor growth, treatment response, and postvascular dormancy. Cancer Res. 59, 4770-4775 (1999)

27. J. Sápi, D.A. Drexler, L. Kovács, Potential benefits of discrete-time controller based treatments over protocol-based cancer therapies. Acta Polytech. Hung. 14(1), 11-23 (2017)

28. P. Baranyi, Extension of the Multi-TP Model Transformation to Functions with Different Numbers of Variables. Complexity 2018 (2018)

29. R. Tóth, Modeling and identification of linear parameter-varying systems, in Lecture Notes in Control and Information Sciences, vol. 403 (Springer, Berlin, 2010)

30. O. Sename, P. Gáspár, J. Bokor, Robust control and linear parameter varying approaches, application to vehicle dynamics, in Lecture Notes in Control and Information Sciences, vol. 437 (Springer, Berlin, 2013)

31. A.P. White, G. Zhu, J. Choi, Linear Parameter Varying Control for Engineering Applicaitons, 1st edn. (Springer, London, 2013)

32. C. Briat, Linear parameter-varying and time-delay systems. Analysis, Observation, Filtering \& Control, 3 (2014)

33. G.B. Thomas, R.L. Finney, M.D. Weir, F.R. Giordano, Thomas' calculus (Addison-Wesley Reading, Boston, 2003)

34. P. Baranyi, TP-model Transformation-based-control Design Frameworks (Springer, Berlin, 2016)

35. L-E. Hedrea, C-A. Bojan-Dragos, R-E. Precup, R-C. Roman, E.M. Petriu, C. Hedrea, Tensor product-based model transformation for position control of magnetic levitation systems, in 2017 IEEE 26th International Symposium on Industrial Electronics (ISIE) (IEEE, 2017), pp. $1141-1146$

36. L-E. Hedrea, C-A. Bojan-Dragos, R-E. Precup, T-A. Teban, Tensor product-based model transformation for level control of vertical three tank systems, in 2017 IEEE 21st International Conference on Intelligent Engineering Systems (INES) (IEEE, 2017), pp. 000113-000118

37. J. Kuti, P. Galambos, P. Baranyi, Minimal volume simplex (MVS) convex hull generation and manipulation methodology for TP model transformation. Asian J. Control 19(1), 289-301 (2017) 
38. P. Galambos, P. Baranyi, TP model transformation: a systematic modelling framework to handle internal time delays in control systems. Asian J. Control 17(2), 1-11 (2015)

39. J. Kuti, P. Galambos, P. Baranyi, Control analysis and synthesis through polytopic tensor product model: a general concept. IFAC-PapersOnLine 50(1), 6558-6563 (2017)

40. S. Campos, V. Costa, L. Tôrres, R. Palhares, Revisiting the TP model transformation: interpolation and rule reduction. Asian J. Control 17(2), 392-401 (2015)

41. Q. Weiwei, H. Bing, L. Gang, Z. Pengtao, Robust model predictive tracking control of hypersonic vehicles in the presence of actuator constraints and input delays. J. Frankl. Inst. 353(17), 4351-4367 (2016)

42. X. Liu, X. Xin, Z. Li, Z. Chen, Near optimal control based on the Tensor-product technique. IEEE Trans. Circuits II 64(5), 560-564 (2017)

43. X. Liu, Y. Yu, Z. Li, H. Iu, Polytopic $\mathrm{H}_{\infty}$ filter design and relaxation for nonlinear systems via tensor product technique. Signal Process. 127, 191-205 (2016)

44. X. Liu, Y. Yu, Z. Li, H. Iu, T. Fernando, A novel constant gain Kalman filter design for nonlinear systems. Signal Process. 135, 158-167 (2017)

45. P.S. Saikrishna, R. Pasumarthy, N.P. Bhatt, Identification and multivariable gain-scheduling control for cloud computing systems. IEEE Trans. Control Syst. Technol. 25(3), 792-807 (2017)

46. Gy. Eigner, I. Böjthe, P. Pausits, L. Kovács. Investigation of the TP modeling possibilities of the Hovorka T1DM model, in 2017 IEEE 15th International Symposium on Applied Machine Intelligence and Informatics (SAMI) (IEEE, 2017), pp. 259-264

47. Gy. Eigner, P. Pausits, L. Kovács, Control of T1DM via tensor product-based framework, in 2016 IEEE 17th International Symposium on Computational Intelligence and Informatics (CINTI) (IEEE, 2016), pp. 55-60

48. Gy. Eigner, I. Rudas, A. Szakál, L. Kovács, Tensor product based modeling of tumor growth, in 2017 IEEE International Conference on Systems, Man, and Cybernetics (SMC) (IEEE, 2017), pp. $900-905$

49. Gy. Eigner, I. Rudas, L. Kovács, Investigation of the tp-based modeling possibility of a nonlinear icu diabetes model, in 2016 IEEE International Conference on Systems, Man, and Cybernetics (SMC) (IEEE, 2016), pp. 3405-3410

50. L Kovács, Gy. Eigner, Convex polytopic modeling of diabetes mellitus: a tensor product based approach, in 2016 IEEE International Conference on Systems, Man, and Cybernetics (SMC) (IEEE, 2016), pp. 003393-003398

51. J. Klespitz, I. Rudas, L. Kovács, LMI-based feedback regulator design via TP transformation for fluid volume control in blood purification therapies, in 2015 IEEE International Conference on Systems, Man, and Cybernetics (SMC) (IEEE, 2015), pp. 2615-2619

52. S. Kuntanapreeda, Tensor product model transformation based control and synchronization of a class of fractional-order chaotic systems. Asian J. Control 17(2), 371-380 (2015)

53. G. Zhao, D. Wang, Z. Song, A novel tensor product model transformation-based adaptive variable universe of discourse controller. J. Frankl. Inst. 353(17), 4471-4499 (2016)

54. W. Qin, B. He, Q. Qin, G. Liu, Robust active controller of hypersonic vehicles in the presence of actuator constraints and input delays, in 2016 35th Chinese Control Conference (CCC) (IEEE, 2016), pp. 10718-10723

55. T. Wang, B. Liu. Different polytopic decomposition for visual servoing system with LMIbased predictive control, in 2016 35th Chinese Control Conference (CCC) (IEEE, 2016), pp. $10320-10324$

56. T. Wang, W. Zhang, The visual-based robust model predictive control for two-DOF video tracking system, in 2016 Chinese Control and Decision Conference (CCDC) (IEEE, 2016), pp. 3743-3747

57. T. Jiang, D. Lin, Tensor product model-based gain scheduling of a missile autopilot. Trans. Jpn. Soc. Aeronaut. Space Sci. 59(3), 142-149 (2016)

58. J. Pan, L. Lu, TP model transformation via sequentially truncated higher-order singular value decomposition. Asian J. Control 17(2), 467-475 (2015) 
59. R.-E. Precup, E.M. Petriu, M.-B. Rădac, S. Preitl, L.-O. Fedorovici, C.-A. Dragoş, Cascade control system-based cost effective combination of tensor product model transformation and fuzzy control. Asian J. Control 17(2), 381-391 (2015)

60. Sz. Nagy, Z. Petres, P. Baranyi, H. Hashimoto, Computational relaxed TP model transformation: restricting the computation to subspaces of the dynamic model. Asian J. Control 11(5), 461-475 (2009)

61. J. Cui, K. Zhang, T. Ma, An efficient algorithm for the tensor product model transformation. Int. J. Control Autom. 14(5), 1205-1212 (2016)

62. A. Szollosi, P. Baranyi, Influence of the tensor product model representation of qLPV models on the feasibility of linear matrix inequality. Asian J. Control 18(4), 1328-1342 (2016)

63. S. Boyd, L. El Ghaoui, E. Feron, V. Balakrishnan, Linear Matrix Inequalities in System and Control Theory, vol. 15. Studies in Applied Mathematics (SIAM, Philadelphia, 1994)

64. K. Tanaka, H.O. Wang, Fuzzy Control Systems Design and Analysis: A Linear Matrix Inequality Approach, 1st edn. (Wiley, Chichester, 2001)

65. M. Chilali, P. Gahinet. $H_{\infty}$ design with pole placement constraints: an lmi approach

66. J.G. VanAntwerp, R.D. Braatz, A tutorial on linear and bilinear matrix inequalities. J. Process. Control 10, 363-385 (2000)

67. P. Gahinet, M. Chilali, P. Apkarian, Robust pole placement in LMI regions. IEEE Trans. Autom. Control 44(12), 2257-2270 (1999)

68. J. Löfberg, Yalmip: a toolbox for modeling and optimization in matlab, in Proceedings of the CACSD Conference, Taipei, Taiwan (2004)

69. MOSEK ApS, The MOSEK optimization toolbox for MATLAB manual. Version 7.1 (Revision 28). (2015)

70. J.K. Tar, J. Bitó, L. Nádai, J.A. Tenreiro Machado, Robust fixed point transformations in adaptive control using local basin of attraction. Acta Polytech. Hung. 6(1), 21-37 (2009)

71. B. Siciliano, L. Sciavicco, L. Villani, G. Oriolo, Robotics-Modelling, Planning and Control, Advanced Textbooks in Control and Signal Processing (Springer, Berlin, 2009)

72. Y. Tagawa, J.Y. Tu, D.P. Stoten, Inverse dynamics compensation via simulation of feedback control systems. Proc. Inst. Mech. Eng. Part I J. Syst. Control Eng. 225(1), 137-153 (2011)

73. H. Musoff, P. Zarchan, Fundamentals of Kalman Filtering: A Practical Approach, 3rd edn. (American Institute of Aeronautics and Astronautics, 2009)

74. J. Hartikainen, A. Solin, S. Särkkä, Optimal Filtering with Kalman Filters and Smoothers a Manual for the Matlab toolbox EKF/UKF. (Aalto University, 2011)

75. L. Kovács, Gy. Eigner, Tensor Product Model Transformation based Par-allel Distributed Control of Tumor Growth. Acta Polytech. Hung. 15(3), 101-123 (2018) 